\title{
Statistical and Computational Evaluation of Axial Loss Correlations Applied to Centrifugal Turbines
}

\author{
Yu Min Liu* \\ Université Libre de Bruxelles, 1050 Brussels, Belgium \\ Patrick Hendrick ${ }^{\dagger}$ \\ Université Libre de Bruxelles, 1050 Brussels, Belgium \\ ZhengPing Zou \\ Beihang University, 100191 Beijing, China \\ Frank Buysschaert ${ }^{\S}$ \\ K.U.Leuven, 1050 Leuven, Belgium
}

\begin{abstract}
Centrifugal turbines have recently regained interest of the engineering community as they could serve as cost-effective alternatives in diverse energy applications. In this device, the energized flow undergoes expansion in the radial outward direction while being entrained by a circular arrangement of airfoil-shaped blades. Yet, their primary and advanced performance estimations have dubiously relied on axial turbine loss correlations as part of their current design paradigm. To the authors knowledge, any convincing and reliable assessment of such practice has still not been reported. Thereupon, this paper conducts an inferential comparative study in which losses of several twin centrifugal and axial cascades are predicted by means of empirical axial loss correlations backed with credible 3D CFD simulations. Ultimately, the significant results disparity and inconsistency points out the inadequacy of the identified practice and the need of a proper and practical centrifugal turbine loss model.
\end{abstract}

\section{Introduction}

The centrifugal turbine has recently been recognized as a cost-effective alternative in small to medium output energy applications encompassing steam [1], ORC [2] and OWC [3] powerplants. Its particularity arises from the expansion of energized centrifugal flow carried out by packs of airfoil-shaped blades arranged in a circular configuration. Due to its approximate resemblance with the axial turbines, their features are commonly compared. For instance, the natural increase of flow area in the centrifugal turbine can accommodate expansion without resort to flowpath flaring as substantiated by the mass conservation. Furthermore, the change of direction of the volume forces cancels the spanwise radial equilibrium that are present in the axial turbines. This implies an enforced $2 \mathrm{D}$ flow in the centrifugal turbine which could greatly simplify the design process.

Yet, its exploitation can be traced back to the beginning of the 19th century with the prominent Ljungström turbine before falling into oblivion for more than half-century [4]. Over the past decades, there are two main research groups [5, 6] that restored this device and provided new design methodologies drawing upon expertise of axial turbines. Although the latter could guide some design choices, there is a targeted practice that raises particular concern [1, 7]. The loss correlations built upon axial turbine flow measurements have been regularly applied to centrifugal turbines in primary and even advanced performance predictions. Historically, Ainley and Mathieson [8] themselves aware of the existence of the Ljungström turbine did not recommend use of their model for the centrifugal design as this would imprudently assume equivalence of both axial and centrifugal flows and it is hardly imaginable that the successive Dunham-Came [9] and Kacker-Ockapuu [10] models could do any exception. With these different perspectives at hand, it is inferred that a thorough investigation on the targeted practice is required.

\footnotetext{
*Ph.D Candidate, Aero-Thermo-Mechanics, yu.m.liu@ulb.ac.be

†Professor, Aero-Thermo-Mechanics, patrick.hendrick@ulb.ac.be

†Professor, School of Energy \& Power Engineering, zouzhengping@ buaa.edu.cn

${ }^{\S}$ Professor, Applied Mechanics \& Energy Conversion, frank.buysschaert@kuleuven.be
} 
Thereby, the present paper assesses the appropriateness of axial loss correlations imprudently applied to centrifugal turbines with inferential statistics. To this end, several subsonic axial turbines are first gathered from open literature and are successively partitioned and transformed into centrifugal cascades with conformal mapping [11] to constitute the samples of the comparative study. Then, the Kacker-Okapuu [10] and Aungier [12] are selected to carry out the near on-design performance prediction of both axial and centrifugal cascades. To back these results, numerical simulations over the same cascades are supplemented utilizing the commercial package FINE/Turbo ${ }^{\mathrm{TM}}$ [13] undergoing a Verification and Validation (V\&V) procedure [14] beforehand. Lastly, a comparison of the samples total pressure losses and deviations is conducted and the significance of the observation is assessed through the T-Test.

\section{Centrifugal Cascades and Statistical Method}

A major challenge in this study is to gather a sufficient amount of centrifugal turbine cases that would form representative samples and prompt reliable statistical analysis. Considering the fact that open literature does not provide any related data, an alternative is opted instead. It consists of creating the centrifugal cascades from available axial turbines by means of conformal mapping and stage partition. This approach brings two properties. The first is the directness that avoids any design process, although this would result in non-optimal centrifugal configuration. The second is the coexistence of twin axial and centrifugal cascades offers a benchmark for a comparative study based on geometrical similitude. With the small size samples at disposition and given the continuous nature of the predicted losses, the comparative study is conducted by means of T-Tests. This effective method reliably assesses the appropriateness of the axial correlations applied to centrifugal turbines by comparing the magnitude of loss disparity of the samples paired units. This section covers both the elaboration of the centrifugal cascades and statistical strategy of the comparative study.

\section{Conformal Mapping}

There are two antagonist similarities that permit a comparative study, i.e. the operating and geometrical similarities. The former must undergo a preliminary design process that for instance enables same velocity triangles for both axial and centrifugal turbines and ineluctably results in distinct blade profiles. On the opposite, the latter which is opted in this study preserves the blade profiles in the centrifugal configuration by means of conformal mapping with no regard for the flow field. In this context, conformal mapping refers to projecting, by means of a $2 \mathrm{D}$ analytic function, the axial blade profile from the axial space to the radial outflow space while preserving the curves intersection angles as depicted schematically in Fig. 1 .
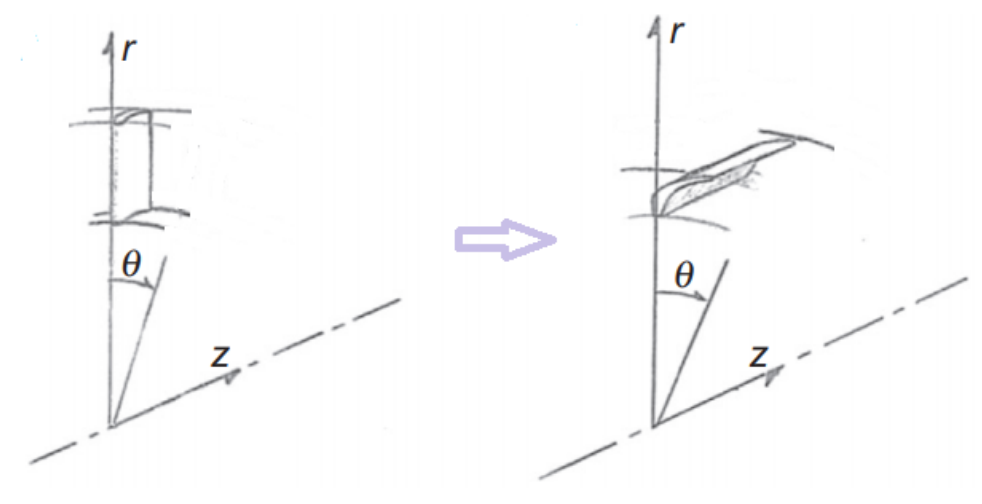

Fig. 1 Conformal transformation from axial to centrifugal configuration.

Its necessity arises from the specificity of the radial outflow configuration in which the radial direction undergoes constant shift along the blade chord to the point of obtaining nonparallel inlet and outlet local axes. In that regard, 
Persico [11] formulated an analytic function to perform the conformal mapping

$$
\begin{aligned}
\log \left(\frac{r_{p}}{r_{i n}}\right) & =\log \left(\frac{r_{\text {in }}}{r_{\text {in }}+b}\right)\left(\frac{x_{p}-x_{\text {ref }}}{b}\right), \\
\theta_{p} & =\log \left(\frac{r_{\text {in }}}{r_{\text {in }}+b}\right)\left(\frac{y_{p}-y_{\text {ref }}}{b}\right) .
\end{aligned}
$$

$\left(x_{p}, y_{p}\right)$ and $\left(r_{p}, \theta_{p}\right)$ are the blade profile coordinates in the axial and radial outflow spaces respectively. $\left(x_{r e f}, y_{r e f}\right)$ is the reference leading edge coordinate. With this relation, the resulting centrifugal blade profile curves maintain the same angles (measured with respect to the radial direction) as the axial ones (measured with respect to the axial direction). Now, the crucial point lies in the selection of the inlet radius $r_{i n}$ for the centrifugal configuration. Persico [11] adjusted $r_{i n}$ to ensure a mid-chord pitch equal to that of axial configuration, completing the geometrical similitude. Despite this, a different approach is advanced and consists of imposing the same inlet operating condition for both twin cascades. This intends to preserve the inlet velocity triangle and is based on the fact that most variable groupings used for turbine performance characterization are generally established upon inlet conditions. With this, $r_{i n}$ is determined by assuming equal blade inlet cross sections and pitch

$$
2 \pi r_{i n} h=\pi\left(r_{\text {tip }}^{2}-r_{\text {hub }}^{2}\right) .
$$

Since $r_{t i p}-r_{h u b}=h$, the resolution of the equation delivers the convenient result $r_{i n}=r_{\text {mean }}$ and induces an increasing pitch along the blade chord. For instance, this yields the blades profiles adjusted to their leading edges as depicted in Fig. 22 The aft is shifted to account the change of radial axis in the centrifugal configuration. Note that if stacking is present in the original axial blade, only the mid-section profile is retained in the transformation.

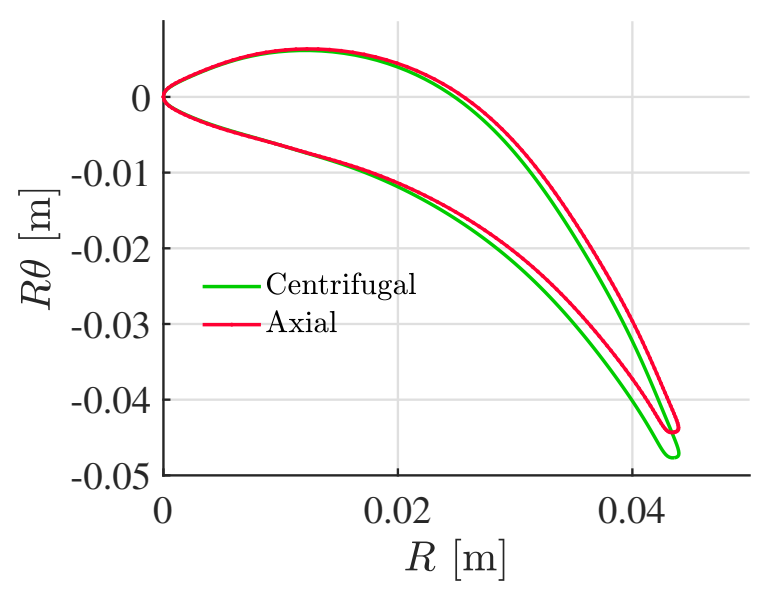

(a) Stator vane.

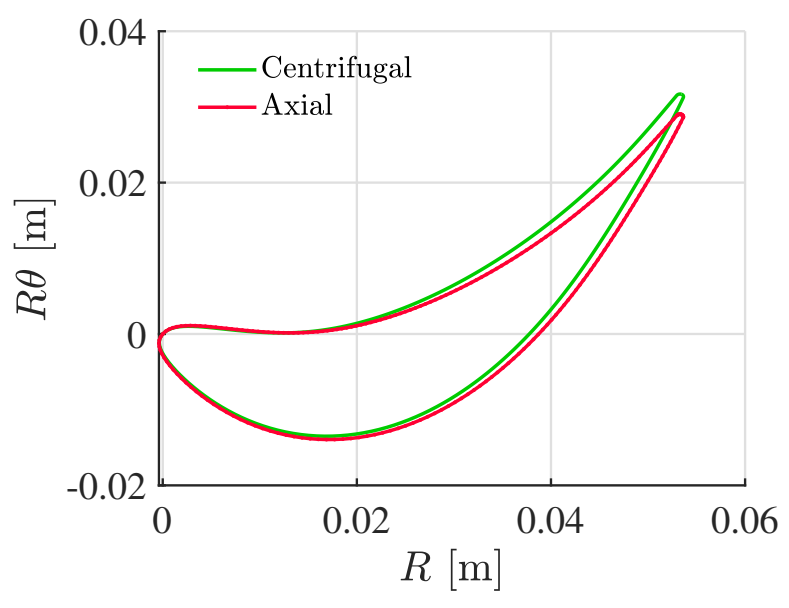

(b) Rotor blade.

Fig. 2 Aachen turbine blade profiles [15] in axial and centrifugal configurations.

The estimate of the velocity field in a centrifugal flow reveals to be particularly useful in the upcoming setup of the boundary conditions and is carried out by means of the notorious radial equilibrium derived from the momentum equation [16]

$$
V_{r} \frac{d V_{r}}{d r}=-\frac{V_{\theta}}{r} \frac{d\left(r V_{\theta}\right)}{d r}
$$

assuming a steady, axisymmetric and isentropic flow and a passage without flaring. The mass conservation yields for the radial velocity component

$$
V_{r}=\frac{\dot{m}}{2 \pi r h \rho} .
$$

By substituting into Eq. 3 , the resolution gives the tangential velocity component

$$
\frac{\left(r V_{\theta}\right)^{2}}{2}-\frac{\left(r_{i n} V_{\theta, i n}\right)^{2}}{2}=\left(\frac{\dot{m}}{2 \pi h \rho}\right)^{2} \log \left(\frac{r}{r_{i n}}\right) .
$$

These relations underscore the diffusion that occurs in the centrifugal passage. 


\section{Turbine Selection}

Despite the limited amount of turbine documentations at disposition, their randomness is assumed, permitting the composition of unbiased and representative samples. To support this, the turbine cases are selected upon diverse and non-redundant sources. Still, only subsonic turbines with removed tip clearance are retained in the selection process. As supersonic and tip leakage flows bring along losses that would unnecessarily complicate the calculation, it is reasonable to discard them from the current study. As result, 12 and 13 annular static and rotating cascades were gathered, covering the range shown in Tab. 1 .

\begin{tabular}{l|cc}
\hline \hline Parameters & Minimum & Maximum \\
\hline$\psi[-]$ & 1.137 & 2.8 \\
$\phi[-]$ & 0.4367 & 0.57 \\
$\eta_{t t}[-]$ & 0.86 & 0.91 \\
$h / c[-]$ & 0.509 & 4.13 \\
\hline \hline
\end{tabular}

Table 1 Design point parameter range of the selected axial turbines.

Even though the conformal mapping is able to preserve the geometry of the blade profiles, the pitch and throat width are extended in the streamwise direction. Aside from the operational parameters, it comes that only the pitch and throat are modified when applying the loss models to the centrifugal cascades as listed in Tab. 2 . More details about the selected cascades can be found in Tab.10

\begin{tabular}{l|cccc}
\hline \hline & \multicolumn{2}{|c}{ Axial } & \multicolumn{2}{c}{ Centrifugal } \\
Parameters & $s / c[-]$ & $o / s[-]$ & $s / c[-]$ & $o / s[-]$ \\
\hline S1 [15] & 0.768 & 0.548 & 0.790 & 0.385 \\
S2 [17] & 0.85 & 0.229 & 0.871 & 0.255 \\
S3 [18] & 0.787 & 0.29 & 0.837 & 0.324 \\
S4 [19] & 0.854 & 0.357 & 0.885 & 0.414 \\
S5 [20] & 0.806 & 0.271 & 0.855 & 0.305 \\
S6 [21] & 0.785 & 0.255 & 0.774 & 0.283 \\
S7 [21] & 0.703 & 0.3387 & 0.717 & 0.396 \\
S8 [22] & 0.499 & 0.496 & 0.511 & 0.544 \\
S9 [22] & 0.606 & 0.427 & 0.613 & 0.454 \\
S10 [23] & 0.634 & 0.344 & 0.678 & 0.395 \\
S11 [24] & 0.862 & 0.562 & 0.883 & 0.597 \\
S12 [24] & 0.706 & 0.440 & 0.718 & 0.458 \\
\hline \hline
\end{tabular}

(a) Twin static cascades.

\begin{tabular}{l|cccc}
\hline \hline & \multicolumn{2}{|c}{ Axial } & \multicolumn{2}{c}{ Centrifugal } \\
Parameters & $s / c[-]$ & $o / s[-]$ & $s / c[-]$ & $o / s[-]$ \\
\hline R1 [15] & 0.697 & 0.459 & 0.716 & 0.528 \\
R2 [17] & 0.81 & 0.319 & 0.822 & 0.349 \\
R3 [25] & 0.85 & 0.327 & 0.864 & 0.357 \\
R4 [25] & 0.93 & 0.33 & 0.960 & 0.350 \\
R5 [18] & 0.711 & 0.339 & 0.823 & 0.355 \\
R6 [19] & 0.813 & 0.409 & 0.882 & 0.476 \\
R7 [20] & 0.784 & 0.68 & 0.806 & 0.414 \\
R8 [21] & 0.835 & 0.375 & 0.824 & 0.411 \\
R9 [21] & 0.753 & 0.524 & 0.813 & 0.528 \\
R10 [22] & 0.683 & 0.493 & 0.695 & 0.503 \\
R11 [22] & 0.711 & 0.454 & 0.722 & 0.468 \\
R12 [26] & 0.840 & 0.388 & 0.851 & 0.407 \\
R13 [24] & 0.857 & 0.634 & 0.879 & 0.681 \\
\hline \hline
\end{tabular}

(b) Twin rotating cascades.

Table 2 Pitch and throat width of the samples

\section{T-Test}

The size of the previously formed samples which is more a constraint than a choice, could incur low statistical power if inappropriate statistical method is used. To cope with this and also guarantee the reliability of the comparative study, an inferential statistical strategy is elaborated.

Firstly, the studied variables have to be defined. Obviously, these would be the total pressure losses and deviations obtained from axial loss correlations and CFD simulations introduced in subsequent sections. As the accuracy of 
the axial correlations is evaluated with benchmark CFD solutions and knowing that there would be inevitable disparities among the solutions of both methods, a fair alternative is advanced and utilizes directly their relative solution difference or error as accuracy indicator

$$
E=\left(S_{M}-S_{N}\right) / S_{N}
$$

Then, given the continuous nature of the variables, the inferential Welsh T-Test [27] is opted as the tool of the comparative study. Its purpose is to reliably compare the means of retained samples variables and determine whether or not there is a trustworthy distinction through a hypothesis test. To guarantee the robustness of the T-Test in the context of small sample analysis, the normality assumption has to be ascertained beforehand [28]. Accordingly, the normal distribution of the samples is respected with the utilization of the subsequent Gaussian AM family loss models [29] and CFD turbulence models [30].

Next, the T-Test comprises a null hypothesis which claims that there is no significant distinction between the means and manifests as a critical P-value of 5\%. In other words, the null hypothesis is set to be respected with a confidence of $95 \%$. In addition, using the T distribution table and prescribing the samples total degree of freedom, the critical P-value is identified by an alternative critical T-value. This T-value is the reference measure in T-tests. Regarding the paired samples, the T-value is obtained with

$$
T=\frac{m_{1}-m_{2}}{s_{p} \sqrt{\frac{1}{N_{1}}+\frac{1}{N_{2}}}}, \quad \text { where } \quad s_{p}=\sqrt{\frac{\left(N_{1}-1\right) s_{1}^{2}+\left(N_{2}-1\right) s_{2}^{2}}{N_{1}+N_{2}-2}} .
$$

$m_{1}, m_{2}, s_{1}, s_{2}$, and $N_{1}, N_{2}$ are the means, standard deviations and sizes of the compared samples respectively. Hence, the observed disagreement between the samples is proven to be statistically significant if the computed T-value is larger than the preset critical T-value, or induces a larger confidence interval than that of the null hypothesis.

Lastly, the comparative study would be divided into two parts. The first aims to determine the axial correlation with superior accuracy based on axial cascades only. By the same occasion, this provides a first assessment of the opted Aungier model [12] that seems to be inexistent in open literature. The second which constitutes the ultimate goal of this study evaluates the validity of the axial correlations applied to centrifugal cascades retaining the axial cascades solution as benchmark. Noting that a particularity of this comparative study is that variables of multiple samples are paired in the statistical sense since they originate from the same source. This raises the study statistical power but at the cost of cutting the effective sample size or degree of freedom by half and thus enforcing stringency of the analysis.

\section{Axial turbine loss and deviation models}

Loss and deviation models have been employed to provide an estimate of turbine performance in early design phase. Those available in open literature essentially differ by application range and accuracy. Yet, two advanced empirical loss and deviation models from the AM family have been selected to carry out the purpose of this work. These are respectively the KO [10], Aungier [12] models. This section outlines their profile and secondary losses and deviation formulations along with the input parameters. The considered pressure loss in absence of blade tip clearance and shock waves takes the form of a linear combination

$$
Y_{t}=\frac{p_{\text {tr }, \text { in }}-p_{\text {tr }, \text { out }}}{p_{\text {tr }, \text { out }}-p_{\text {out }}}=Y_{p}+Y_{s}+Y_{\text {te }} .
$$

These loss components are assumed to have weak mutual interactions and thus enable superposition. Nevertheless, a specific adjustment to $r_{\text {in }}$ is carried out when applying Eq. 8 on the centrifugal cascade as blade speed increases with radius.

\section{Kacker \& Okapuu model}

The KO model [10] is a prominent model of the AM family and is still extensively utilized nowadays. Chronologically, the AM model [8] was established upon fifties conventional turbine cascade flow measurements and could achieve a standard precision of $\pm 2 \%$ for efficiency prediction. The DC model [31] updated the AM model with seventies technology turbine cascade flow measurements and extended its application range especially to accommodate small turbines, while maintaining the same precision for efficiency prediction. Owing to the success of these models, the KO 
model came as a second update by means of tests over 33 eighties technology turbine stages and could improve the standard precision up to $\pm 1.5 \%$ for efficiency prediction near design point.

Its profile loss is defined as

$$
Y_{p}=0.914 K_{R e}\left(K_{\text {mod }} K_{p} K_{M} K_{\text {inc }} Y_{p, A M}+Y_{\text {sh }}\right) .
$$

The constant 0.914 corrects the profile loss to zero trailing edge thickness because the trailing edge loss is treated separately. Unlike the AM and DC models, the Reynolds number correction $K_{R e}(R e)$ only applies to the profile loss and is triggered beyond the range $R e \in\left[2 \times 10^{5}-10^{6}\right]$. The technology factor $K_{\text {mod }}$ taking the value of $2 / 3$ adjusts the over-conservative profile loss $Y_{p, A M}$ to modern turbines. For subsonic high velocity flow, compressibility affects the profile loss through $K_{p}\left(M_{r, \text { in }}, M_{r, \text { out }}\right)$ and $Y_{\text {sh }}\left(p_{\text {in }}, p_{\text {out }}, M_{r, h u b, \text { in }}, M_{r, \text { in }}, M_{r, \text { out }}, d_{t i p}, d_{h u b}, k\right)$. The former characterizes the beneficial inter-blade channel flow acceleration that prevents boundary layer buildup. The latter accounts local shocks caused by utilization of thicker leading edge at the hub section and $M_{r, \text { hub, in }}>0.4 . K_{M}\left(M_{r, \text { out }}\right)$ is an arbitrary and dubious factor introduced by DC to cover the over-expansion at the aft portion of the blade in case of supersonic flow with $M_{r, \text { out }}>1$. The incidence correction $K_{\text {inc }}\left(i, s / c, \theta_{\text {in }}, \beta_{\text {out }}\right)$ is identical to that in Ref. [8]. The original profile loss $Y_{p, A M}$ without incidence is expressed as

$$
Y_{p, A M}=\left[Y_{p, \theta_{\text {in }}=0^{\circ}}-\left|\frac{\theta_{\text {in }}}{\beta_{\text {out }}}\right|\left(\frac{\theta_{\text {in }}}{\beta_{\text {out }}}\right)\left(Y_{p, \theta_{\text {in }}=-\beta_{\text {out }}}-Y_{p, \theta_{\text {in }}=0^{\circ}}\right)\right]\left(\frac{5 t_{\text {max }}}{c}\right)^{\frac{-\theta_{\text {in }}}{\beta_{\text {out }}}},
$$

which blends the profile losses acquired on contrasting nozzle $\left(\theta_{\text {in }}=0^{\circ}\right)$ and impulse $\left(\theta_{\text {in }}=-\beta_{\text {out }}\right)$ blades. It is interesting to note that the profile loss of the AM family is actually not applicable to blades with low deflection such that $\theta_{\text {in }} / \beta_{\text {out }}>0$, as Eq. 10 becomes negative [32].

The secondary loss is

$$
Y_{S}=1.2 K_{p} K_{A R} Y_{S, A M D C} .
$$

Again, the constant 1.2 is prescribed to correct the secondary loss to zero trailing edge thickness. The aspect ratio factor $K_{A R}(h / c)$ was first introduced by DC and was readjusted to prevent excessive shooting at $h / c \leqslant 2$. The original secondary loss $Y_{S, A M D C}$ is

$$
Y_{s, A M D C}=0.0334\left(\frac{\cos \beta_{\text {out }}}{\cos \theta_{\text {in }}}\right) Z
$$

The constant 0.0334 conveniently and crudely represents the effect of upstream wall boundary layer. The Ainley loading parameter $Z\left(\beta_{\text {in }}, \beta_{\text {out }}, s / c\right)$ corresponds to an alternative interpretation of the blade loading.

As surprising as it may be, the deviation associated to either DC or KO models is still the one originally suggested by AM [8]. The AM deviation model firstly assumes that the outlet gas angle is independent of inlet incidence. This generally applies to thick leading edge designs or confines the operation range to near design point. Then in the low subsonic range identified by $M_{r, \text { out }} \in[0-0.5]$, the outlet gas angle is independent of the exit Mach number such that

$$
\beta_{\text {out }}=f\left(\arccos \frac{o}{s}\right)-4\left(\frac{s}{e}\right),
$$

where $f(\arccos o / s)$ is a function established upon straight back profile cascade flow measurement. Next, it is deduced that once the sonic state $M_{r, \text { out }}=1$ is reached, the exit angle becomes

$$
\beta_{\text {out }}=-\arccos \frac{A_{o}}{A_{\text {out }}} .
$$

Eventually in the high subsonic range with $M_{r, \text { out }} \in[0.5-1]$, a linear interpolation between Eq. 13 and Eq. 14 with $M_{r, \text { out }}$ is performed.

The KO model sample encompasses the range summarized in Tab 3 and it can be seen that the selected turbine cases falls within the application range of the KO model by referring to Tab.1.

Still, there is an additional limitation of the KO model that is worth disclosing and actually justifies the need of the supplementary Aungier model. As underscored by the authors, the model is restricted to competent designs which omit 


\begin{tabular}{l|cc}
\hline \hline Parameters & Minimum & Maximum \\
\hline$\psi[-]$ & 1.02 & 2.71 \\
$\phi[-]$ & 0.309 & 1.5 \\
$\eta_{t t}[-]$ & 0.78 & 0.93 \\
$h / c[-]$ & 0.24 & 5.8 \\
\hline \hline
\end{tabular}

Table 3 Design point parameter range of the axial turbines in the KO model [10].

recompression at any blade section. This feature has been inherited from the AM model and makes it unsuitable for performance analysis of low reaction turbines. As reported by Lozza [32], the latter could exhibit local recompression manifesting as unacceptable overshoot of secondary loss at off-design conditions.

Besides the efficiency accuracy, it is also of interest to gain an insight on the loss prediction accuracy that would affect the interpretation of the upcoming results analysis. Surprisingly, there are only few references that addresses this topic in details. Wei [33] and Benini et al. [34] provided a comparison between the measured loss on a low aspect ratio two stages turbine rig and several loss model predictions. Among these, the KO model could overestimate up to $33 \%$ and $200 \%$ above the stator and rotor benchmark values respectively and it is inferred by loss breakdown that the secondary loss has the largest contribution at near design conditions. However, this study lacks representativeness as a single test case is evaluated. Benner et al. [35] observed with 34 diversified cascade tests that the KO secondary loss could rise up to $500 \%$ above the measured loss and advanced the need of an engine-to-cascade scaling factor of 0.23 to match the data. Nevertheless, this constitutes a single observation only and should have been finalized with adequate statistical methods to enhance the study's potency. Baturin et al. [29] established a 95\% confidence interval of $\pm 146 \%$ width and around a $20 \%$ mean profile loss disparity with a large dataset comprising 170 linear cascades. Altogether, these sources indicate that the KO secondary loss is likely to cause the largest disparity in a typical loss comparison. Nevertheless, it is worthy to note that two third of the KO model sample are made up of high pressure turbines manufactured by Pratt\&Whitney. As these share the same technology, the redundancy and also lack of randomness could bias the model, thus providing a possible explanation for the large disparity observed on non-Pratt\&Whitney designs.

\section{Aungier model}

The Aungier model [12] is the successor of the KO model and broadens its application range to low reaction turbines by partly imitating the $\mathrm{CC}$ model [36] and real fluid turbines as demonstrated by $\mathrm{Da}$ Lio et al. [37]. It essentially revises the KO model by essentially bringing appropriate reformulation of factors intervening in the profile and secondary losses in order to prevent overshooting at extreme off-design conditions.

The profile loss is defined as

$$
Y_{p}=K_{\text {mod }} K_{\text {inc }} K_{M} K_{p} K_{R e}\left(Y_{p, A M}-Y_{t e=0.02 s}\right) .
$$

Contrary to Eq.9 the correction to zero trailing edge thickness is done by subtraction of $Y_{t e=0.02 s}$. Nevertheless, $K_{\text {mod }}$ and $K_{i n c}$ are kept identical to those in Eq.9 $K_{R e}$ is modified to account the effect of surface finish beyond $R e>5 \times 10^{5}$. Like the DC model, it is reapplied to the secondary loss. $K_{M}\left(M_{r, \text { out }}, s, e\right)$ is fundamentally redefined and is extended to include local expansion loss in transonic band $M_{r, \text { out }} \in[0.6-1]$. The significant contribution of Aungier lies in $K_{p}\left(M_{r, \text { in }}, M_{r, \text { out }}\right)$ which can now encompass the aforementioned recompression at any operating condition.

The secondary loss is written as

$$
Y_{S}=K_{p} K_{R e}\left(\frac{K_{A R}^{2} Y_{s, A M D C}^{2}}{1+7.5 K_{A R}^{2} Y_{s, A M D C}^{2}}\right)^{\frac{1}{2}} .
$$

The square root formulation has the purpose to prevent overshoot at extreme off-design conditions as mentioned earlier. $K_{A R}(h / c)$ is of same nature as that of the KO model but is slightly lowered for low aspect ratio blades.

The Aungier model comprises its own deviation model and provides directly the deviation instead of the gas outlet angle. Similarly to the AM model, the deviation is independent of the discharge Mach number in the low subsonic 
range $M_{r, \text { out }} \in[0-0.5]$ and is expressed as

$$
\delta=\arcsin \left(\left(\frac{o}{s}\right)\left[1+\left(1-\frac{o}{s}\right)\left(\frac{\arcsin o / s}{90}\right)^{2}\right]\right)-\arcsin o / s .
$$

Note that the presence of arcsin instead of arccos is due to a different reference system used by Aungier. In the high subsonic range $M_{r, \text { out }} \in[0.5-1]$, a fifth degree polynomial is implemented

$$
\delta=\delta_{E q .17}\left[1-10\left(2 M_{r, \text { out }}-1\right)^{3}+15\left(2 M_{r, \text { out }}-1\right)^{4}-6\left(2 M_{r, \text { out }}-1\right)^{5}\right] .
$$

Regardless of the input throat to pitch ratio $o / s$, this function cancels when sonic state $M_{r, \text { out }}=1$ is reached.

The inputs of the outlined factors intervening in each loss and deviation would be obtained with the subsequent CFD simulations. Additionally, it is evident that the axial correlations would not be insensitive to the centrifugal flow that alters in the outlet operational input parameters.

\section{Numerical Method}

Since the numerical solutions serve as benchmark to assess the accuracy of the axial correlations, an emphasis is given to the elaboration of a rather comprehensive CFD strategy. Despite the prevalence of CFD gained over the past decades in many fields of study and industries, it is subject to limitations. Mainly, the latter arises from the overriding dependence of the computed solution upon the numerical discretization and turbulence modelling [38]. Without prior verification and validation of the opted CFD strategy, any produced solutions would be untrustworthy [39]. In this regard, the systematic V\&V approach developed at the University of Iowa [14, 40] is utilized. It begins with the description of the CFD strategy encompassing the EURANUS code, boundary conditions, assumptions, grid properties and turbulence models. Successively, verification and validation are conducted on the Aachen turbine rotor [41] for which there are abundant experimental measurements [15] and thereby the grid and turbulence model best suited for the simulation of the previously gathered cascades are determined.

\section{Space and Time Discretization Strategies}

The solver EURANUS [42] part of the package Fine/Turbo ${ }^{\mathrm{TM}}$ solves the weak and conservative form of the Favre/Reynolds-Averaged Navier-Stokes (RANS) equations, basing on cell-centered finite volume method. The inviscid convection and viscous diffusion terms are both discretized by central difference. However, the former is not compatible with the opted discretization scheme and thus requires moderate damping that prevents onset of spurious oscillations within the mean-flow solution while preserving higher order resolution [43]. In this regard, central artificial dissipation [44] using STVD technique [45] was supplemented.

The steady solution was achieved through time marching which involves time integration with the explicit five stage second order Runge-Kutta scheme. Furthermore, to guarantee numerical stability, a reasonable CFL $=3$ has been prescribed. The convergence rate has been accelerated by applying the local time stepping, implicit residual smoothing [46] and FAS multigrid strategy. Regarding the latter, the high frequency part of the solution is smoothed over a three to four grid levels V-cycle. Yet, time marching algorithms designed for compressible flows exhibit a pronounced lack of efficiency in terms of convergence rate and round off errors in low Mach number flows. To correct these disruptions, the Merkle preconditioning [47] is employed. In sum, the code architecture is capable of maximum second order accuracy.

\section{Turbulence Modelling}

To raise the odds of the upcoming validation, two distinct RANS turbulence closure models were considered. The first is the prominent isotropic SST model established by Menter [48] as an extension to the BSL model [49]. These rely on an isotropic Reynolds stress tensor assuming the Boussinesq approximation and introduces two additional transport equations for the turbulent kinetic energy and length or time scale through the eddy viscosity. The BSL model blends the $k-\omega[50]$ and the $k-\epsilon$ [51] models utilizing a zonal approach that fully exploits the best of both models. In fact, it comprises a switch function that activates the $k-\omega$ model in the near-wall region and the $k-\epsilon$ model in the outer wake region and in free shear layers respectively. The additional feature of the SST model is the modification to eddy viscosity 
basing on the Bradshaw assumption [52] and thereupon exhibits better behavior under adverse pressure gradient flow.

The second model arises from the inability of the isotropic models to capture essential properties such as the inherent anisotropy of turbulence, impact of body forces on turbulence [53]. To remedy these shortfalls, the promising S-EARSM model [54] derived from the WJ-EARSM model [55] is opted. It fundamentally consists of incrementing an anisotropic normal stress tensor defined as a quadratic combination of strain and vorticity rate tensors to the isotropic Reynolds stress tensor. The turbulent transport equations are treated in the same way as in the BSL model to permit similar behavior against the SST model for shear flows in which anisotropy does not intervene. Although this model reveals to be more influential in applications involving severe separation [56, 57], its asset in this context lies in the ability to exhibit the body forces effect on turbulence brought by the theoretical augmentation.

The turbine cases are designed with transitional range Reynolds numbers. In that respect, the blade boundary layer transition could potentially cover up to $10 \%$ of the chord length [58] and could thus significantly alter the profile loss. The opted SST and S-EARSM models can exhibit apparent transition, but this actually suffers from inconsistency caused by sensitivity to grid resolution [59]. With these in mind, it is necessary to couple the turbulence models with a proper transition model. In few words, the transition model involves two transport equations for intermittency and momentum thickness Reynolds number which respectively triggers transition locally and captures the local influence of turbulence intensity. Literature offers plenty of empirical transition models, each having its own specific range of application [60]. Among these, the model of Kelterer et al. [61] which corresponds to a calibrated version of Langtry \& Menter [62] $\gamma-\operatorname{Re}_{\theta}$ model to the turbomachinery flow is adopted.

\section{Boundary Conditions and Assumptions}

Substantial computational expense was spared by exploiting the periodicity of each row. Absolute total temperature and pressure profiles were imposed at the inlet patch placed at one chord upstream of the leading edge. Averaged static pressure was subsequently been prescribed to enhance the global algorithm robustness, at the outlet patch placed at one chord downstream of the trailing edge. Periodic boundary conditions have been imposed on the circumferential patches of the control volume. In order to reproduce the experimental flow in the $\mathrm{V} \& \mathrm{~V}$ procedure, the experimental conditions of Tab. 3 are imposed at the boundary patches of the control volume.

Steady simulations were opted in this framework for their capability of providing the steady part of the flow solution as a first order approximation at lowest computational cost. It is worth noting that the steady and time averaged quantities are not comparable in the strict sense. Nonetheless, practice could tolerate the comparison if the absence of time fluctuation in the flow solution is assumed negligible.

The input quantities of the loss models are retrieved at $2 \%$ meridional chord upstream and downstream of the blade leading and trailing edges respectively. This unusual proximity prevents the diffusion of the centrifugal flow which alters the quantities with distance apart as reported by Persico [63].

Complying with the V\&V approach [14], the resulting uncertainty associated to the simulation is the sum of two principal contributions, i.e. the numerical and modelling uncertainties,

$$
U_{S}^{2}=U_{S N}^{2}+U_{S M}^{2}+U_{S P D}^{2}
$$

The upcoming verification specifically addresses $U_{S N}$ decomposed into iterative, grid spacing and time step uncertainties

$$
U_{S N}^{2}=U_{I}^{2}+U_{G}^{2}+U_{T}^{2} \approx U_{G}^{2}
$$

By ensuring a global iterative residual of about $10^{-5}$ or lower upon reaching convergence, it is reasonable to assume a negligible $U_{I}$ compared to to other contributions, as shown in the examples of Ref. [64]. $U_{T}$ is naturally discarded in the context of steady simulations as it intervenes in the false transient that is quickly damped by the configured time discretization strategy. At last, verification is aimed at the estimation of $U_{G}$. As for the validation, a similar approach is utilized and determines the comparison uncertainty or noise level defined as

$$
U_{E}^{2}=U_{D}^{2}+U_{S N}^{2}+U_{S M}^{2}+U_{S P D}^{2},
$$


where $U_{D}$ and $U_{S P D}$ correspond to the uncertainties associated to the test repeatability at a given operating condition and numerical output determined from experimental data respectively. As Stern et al. [40] did not provide any practical mean to quantify $U_{S P D}$, a plausible approximation would be that the CFD code is insensitive to the boundary conditions uncertainty such that

$$
U_{D}=U_{S P D}
$$

Thereby, the numerical solution straightforwardly inherits the uncertainty of the experimental data. Validation also depends on the comparison error defined as the difference between the experimental and numerical solutions

$$
E=D-S \text {. }
$$

\section{Grid Properties}

For an effective grid generation, there are three aspects to pay attention to, i.e. grid type, quality and density. Now as surprising as it may be, the effect of the grid type and quality on the numerical accuracy is still not clearly grasped [65]. The grid type and quality ratings could solely rely on some theoretical groundwork and few guidelines based on past experience. From a practical standpoint and by comparison to other existing grid varieties, hexahedral grids produce results of better numerical accuracy for RANS simulations of turbine flows [66] and have thus been adopted in this framework. Semi-automatic grid generation has been conducted by means of AutoGrid5 ${ }^{\mathrm{TM}}$. The latter exploits the composite multi-block strategy which notably permits local refinement of the grid resolution around the blade profiles. A O4H topology (visible over the hub in Fig. 4a which comprises batches of curvilinear structured hexahedral blocks, is opted. Wall flow simulations are computationally demanding and necessitate positioning of first wall cells within the viscous sublayer defined by non-dimensional wall distances $y^{+}<5$ [48]. For this purpose, large portion of cells are clustered at the wall boundaries and plane intersections to enable first inner cell spacing characterized by $y^{+} \approx 0.8$ (Fig. 4b]. The geometric wall distance is estimated with the Blasius formula [13] and rotor exit condition

$$
y=6 y^{+}\left(\frac{V_{\text {out }}}{v}\right)^{-7 / 8}\left(\frac{c}{2}\right)^{1 / 8} .
$$

Furthermore, uncontrolled cell size variation and geometric distortion referred as grid quality deterioration, could introduce significant parasitic term that magnifies the discretization error of the numerical scheme [67]. To prevent this in the near-wall region, cell orthogonality and expansion ratio of 1.2 have been enforced on the wall cells.

\section{Test Case : Aachen 1.5 Stage Turbine}

Presented in the framework of ERCOFTAC [15], the RWTH Aachen 1.5 stage turbine test rig was built with the aim of studying secondary flows either experimentally or numerically [41, 68]. The stators and rotor (Fig. 3] are respectively equipped with the Traupel [69] and modified VKI profile blades.

Strictly speaking, the CFD code reliability could only be confirmed by executing the V\&V procedure on all the turbines exposed in Tab. 10 However, this would constitute a tedious task that does not bring any apparent added values to this study. Instead, considering the wide operating conditions (Fig. 33 of this particular turbine and related experimental data at hand, the $\mathrm{V} \& \mathrm{~V}$ procedure is narrowed to a single test case. Aubé and Hirsch [70] also conducted a study on this turbine to exhibit the capability of FINE/Turbo ${ }^{\mathrm{TM}}$ in the past. They showed the possibility to obtain steady results matching with the experimental data by deliberately adjusting the outlet static pressure of the numerical model although that would depart from the actual experimental test conditions. Since the body forces are crucial in this work, they only manifest themselves in the rotor. Thereby, it would be judicious to further narrow the $\mathrm{V} \& \mathrm{~V}$ procedure to the turbine rotor row only on which the experimental conditions are imposed. 


\begin{tabular}{l|cc}
\hline \hline Operating condition & $\mathrm{A}$ & $\mathrm{B}$ \\
\hline$p_{t, \text { in }}[\mathrm{Pa}]$ & 155055.45 & 171022.11 \\
$T_{t, \text { in }}[\mathrm{K}]$ & 308.20 & 306.11 \\
$p_{\text {out }}[\mathrm{Pa}]$ & 110050.96 & 110562.52 \\
$\dot{m}[\mathrm{~kg} / \mathrm{s}]$ & 6.8395 & 8.0205 \\
$\Omega[\mathrm{RPM}]$ & 3496.47 & 3503.82 \\
$\psi[-]$ & 1.1957 & 1.639 \\
$\phi[-]$ & 0.4367 & 0.5288 \\
$\operatorname{Re}[-]$ & $5 \times 10^{5}$ & $5.36 \times 10^{5}$ \\
\hline \hline
\end{tabular}

(a) Operating conditions.

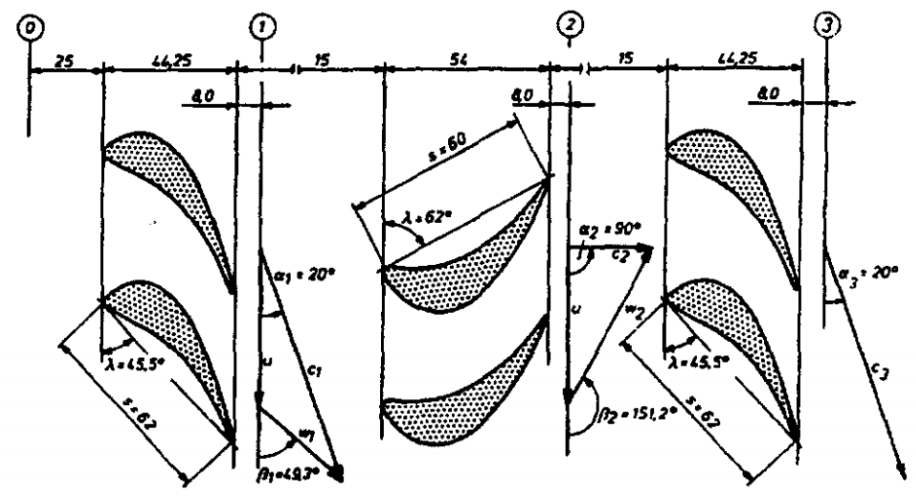

(b) Geometry.

Fig. 3 Features of the Aachen turbine [41].

Three grids of distinct densities were generated beforehand for the upcoming verification and are listed in Tab. 4 . Baker [65] suggests a consistent grid refinement characterized by a ratio $r_{G}=2$ to expose the sensitivity of the flow solution to the grid resolution. Note that consistent grid refinement applies to each space coordinate, is constant and not a function of space and time [71].

\begin{tabular}{l|ccc}
\hline \hline Grid & G1 & G2 & G3 \\
\hline$y$ [m] & $1.28 \times 10^{-5}$ & $6.4 \times 10^{-6}$ & $3.2 \times 10^{-6}$ \\
O-grid nodes [-] & $9 \times 35 \times 72$ & $17 \times 69 \times 145$ & $33 \times 137 \times 289$ \\
Tip clearance nodes [-] & $5 \times 9 \times 72$ & $13 \times 17 \times 145$ & $25 \times 33 \times 289$ \\
Rotor row nodes [-] & $4.72 \times 10^{4}$ & $3.38 \times 10^{5}$ & $2.56 \times 10^{6}$ \\
\hline \hline
\end{tabular}

Table 4 Grid specification of the Aachen turbine rotor row.

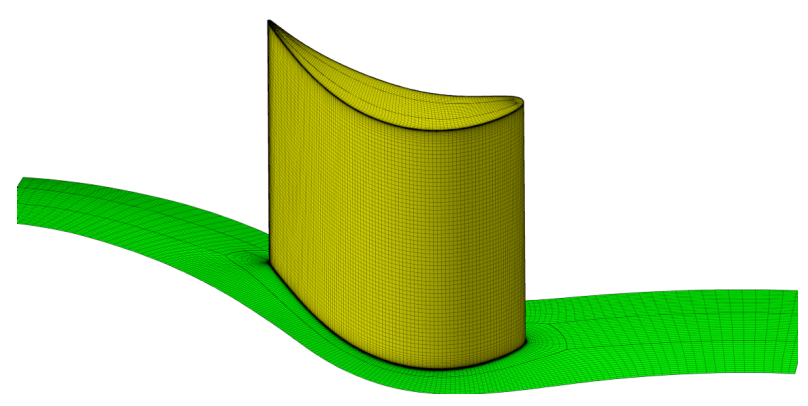

(a) Meshing topology

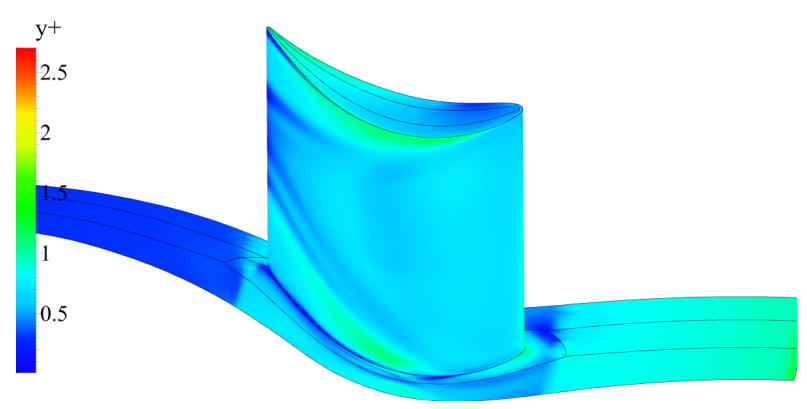

(b) Nondimensional wall distance.

Fig. 4 Grid 3 of Aachen turbine rotor blade.

\section{Verification}

Verification is defined as a procedure that deals only with the accuracy of the solution of the assumed mathematical model and quantifies the uncertainty of all possible numerical error sources [39]. Since the latter was reduced to the sole spatial discretization uncertainty in Eq.20, the procedure would now consist of a sensitivity analysis, i.e. the generalized Richardson extrapolation (RE), conducted on three systematically refined grids shown in Tab. 4 Nonetheless, to enable the sensitivity analysis, monotonicity of the grid solutions has to be proven beforehand using the ratio

$$
R_{G}=\frac{\epsilon_{23}}{\epsilon_{12}}=\frac{S_{G 2}-S_{G 3}}{S_{G 1}-S_{G 2}} .
$$


This is actually inspired from the monotonicity related to the numerical scheme limiters and prevents under- or over-shooting of successive solution gradients [43]. Monotonicity is achieved with $\left.R_{G} \in\right] 0-1[$. In case of divergence, verification is aborted. Either the opted discretization strategies produced large unexpected numerical errors spoiling the nature of the RANS equations or the grids were ill-defined. If monotonicity is met, then assuming that the grid solutions are close to an asymptote and thus differ by little variation, RE delivers an estimate of the numerical error and the order of accuracy related to spatial discretization

$$
\begin{gathered}
\delta_{R E}=\frac{\epsilon_{23}}{r_{G}^{p_{G}}-1}, \\
p_{G}=\frac{\log \left(\epsilon_{12} / \epsilon_{23}\right)}{\log \left(r_{G}\right)} .
\end{gathered}
$$

$\mathrm{RE}$ is inappropriate in most cases since the proximity of the solutions to an asymptote is unknown and can not be confirmed in advance. To remedy this, Stern et al. [40] introduced an additional correction factor defined as

$$
C_{G}=\frac{\left(\epsilon_{12} / \epsilon_{23}-r_{G}^{q_{G, e s t}}\right)\left(r_{G}^{p_{G}}-1\right)}{\left(r_{G}^{p_{G, e s t}}-r_{G}^{q_{G, e s t}}\right)\left(r_{G}^{p_{G, e s t}}-1\right)}+\frac{\left(\epsilon_{12} / \epsilon_{23}-r_{G}^{p_{G, e s t}}\right)\left(r_{G}^{p_{G}}-1\right)}{\left(r_{G}^{p_{G, e s t}}-r_{G}^{q_{G, e s t}}\right)\left(r_{G}^{q_{G, e s t}}-1\right)},
$$

for $p_{G} \leq 1$ and $p_{G, e s t}=1$ and $q_{G, e s t}=2$ are initial estimates of the order of accuracy. Instead, the following formulation is given for $p_{G}>1$

$$
C_{G}=\frac{r_{G}^{p_{G}}-1}{r_{G}^{q_{G, e s t}}-1} .
$$

This serves as measure of confidence and also as scale factor to $\delta_{R E}$ and if $C_{G} \approx 1$, then the grid solutions are effectively following an asymptote and the results produced by RE are correct and trustworthy. Regardless of the outcome, the spatial discretization uncertainty is written as

$$
U_{G}= \begin{cases}{\left[9.6\left(1-C_{G}\right)^{2}+1.1\right]\left|\delta_{R E}\right|} & :\left|1-C_{G}\right|<0.125 \\ {\left[2\left|1-C_{G}\right|+1\right]\left|\delta_{R E}\right|} & :\left|1-C_{G}\right| \geq 0.125\end{cases}
$$

This function possesses a minimum at $C_{G}=1, \forall \delta_{R E}$. This means that uncertainty arises from the remoteness of the grid solutions from the asymptotic range. Note that the described procedure could also cover the uncertainties related to time step, artificial dissipations and so on.

The verified flow quantities are selected upon the essential variables intervening in the axial loss correlations. These are the static and total pressures, Mach number and gas angle. Tab. 5 and 6 gather the values acquired at the rotor exit plane depicted in Fig. 3 with both SST and S-EARSM turbulence models.

\begin{tabular}{l|cccccccc}
\hline \hline Variable & $S_{G 3}^{-}$ & $\epsilon_{12}\left[\% S_{G 3}^{-}\right]$ & $\epsilon_{23}\left[\% S_{G 3}^{-}\right]$ & $R_{G}$ & $p_{G}$ & $E_{R E}\left[\% S_{G 3}^{-}\right]$ & $C_{G}$ & $U_{S N}\left[\% S_{G 3}^{-}\right]$ \\
\hline$p_{A}[\mathrm{~Pa}]$ & $1.302 \times 10^{5}$ & 0.3 & 0.22 & 0.726 & 0.462 & 0.59 & 0.534 & 1.13 \\
$p_{\text {tr }, \text { out }, A}[\mathrm{~Pa}]$ & $1.39 \times 10^{5}$ & 2.25 & 0.75 & 0.334 & 1.584 & 0.38 & 0.666 & 0.63 \\
$\beta_{\text {out }, A}\left[{ }^{\circ}\right]$ & 65.4 & 14.8 & 6.8 & 0.459 & 1.123 & 5.77 & 0.393 & 12.78 \\
$M_{r, \text { out }, A}[-]$ & 0.304 & 19.95 & 6.45 & 0.323 & 1.629 & 3.08 & 0.698 & 4.94 \\
\hline$p_{B}[\mathrm{~Pa}]$ & $1.401 \times 10^{5}$ & 0.51 & 0.31 & 0.596 & 0.746 & 0.45 & 0.823 & 0.61 \\
$p_{\text {tr }, \text { out }, B}[\mathrm{~Pa}]$ & $1.499 \times 10^{5}$ & 2.43 & 1 & 0.412 & 1.277 & 0.7 & 0.475 & 1.44 \\
$\beta_{\text {out }, B}\left[{ }^{\circ}\right]$ & 66.03 & 17.74 & 7.65 & 0.431 & 1.214 & 5.8 & 0.44 & 12.29 \\
$M_{r, \text { out }, B}[-]$ & 0.307 & 19.51 & 8.04 & 0.412 & 1.279 & 5.64 & 0.475 & 11.55 \\
\hline \hline
\end{tabular}

Table 5 Verification at rotor exit plane using SST $\gamma-R e_{\theta}$. 


\begin{tabular}{l|cccccccc}
\hline \hline Variable & $S_{G 3}^{-}$ & $\epsilon_{12}\left[\% S_{G 3}^{-}\right]$ & $\epsilon_{23}\left[\% S_{G 3}^{-}\right]$ & $R_{G}$ & $p_{G}$ & $E_{R E}\left[\% S_{G 3}^{-}\right]$ & $C_{G}$ & $U_{S N}\left[\% S_{G 3}^{-}\right]$ \\
\hline$p_{A}[\mathrm{~Pa}]$ & $1.302 \times 10^{5}$ & 0.22 & 0.1 & 0.453 & 1.14 & 0.08 & 0.403 & 0.18 \\
$p_{\text {tr }, \text { out }, A}[\mathrm{~Pa}]$ & $1.39 \times 10^{5}$ & 2.39 & 0.68 & 0.285 & 1.813 & 0.27 & 0.837 & 0.36 \\
$\beta_{\text {out }, A}\left[{ }^{\circ}\right]$ & 63.49 & 13.84 & 4.36 & 0.318 & 1.668 & 2 & 0.726 & 3.1 \\
$M_{r, \text { out }, A}[-]$ & 0.299 & 21.17 & 5.98 & 0.282 & 1.824 & 2.35 & 0.847 & 3.08 \\
\hline$p_{B}[\mathrm{~Pa}]$ & $1.40 \times 10^{5}$ & 0.26 & 0.1 & 0.389 & 1.361 & 0.06 & 0.523 & 0.13 \\
$p_{\text {tr }, \text { out }, B}[\mathrm{~Pa}]$ & $1.496 \times 10^{5}$ & 2.52 & 0.76 & 0.303 & 1.721 & 0.33 & 0.766 & 0.49 \\
$\beta_{\text {out }, B}\left[{ }^{\circ}\right]$ & 63.56 & 13.54 & 4.86 & 0.359 & 1.477 & 2.73 & 0.595 & 4.94 \\
$M_{r, \text { out }, B}[-]$ & 0.301 & 20.82 & 6.22 & 0.299 & 1.743 & 2.65 & 0.782 & 3.81 \\
\hline \hline
\end{tabular}

Table 6 Verification at rotor exit plane using S-EARSM $\gamma-R e_{\theta}$.

First, monotonicity of the numerical solutions is unanimously guaranteed. Next, the variables have more or less consistent results at the exception of $p$ and also $\beta$. The severely lower order of accuracy of $p$ is due to the nature of the flow domain exit boundary condition that imposes the static pressure and constrains the flow computation. The mild issue of $\beta$ arises from the flow treatment at the wall boundary conditions upon which the velocity components are cancelled and the flow angle is set to a default value of $\pi / 2$. Lastly, a deterioration of the numerical resolution is observed at off-design operating condition. According to the entropy contours depicted in Fig. 5. important flow separation is triggered at the blade aft. This prompts severe physical dissipation but also numerical dissipation, thus spoiling the numerical order of accuracy. Overall, the SST model exhibits accrued disparity and susceptibility than its S-EARSM counterpart, leading to lower confidence in the numerical solutions and larger numerical uncertainty.

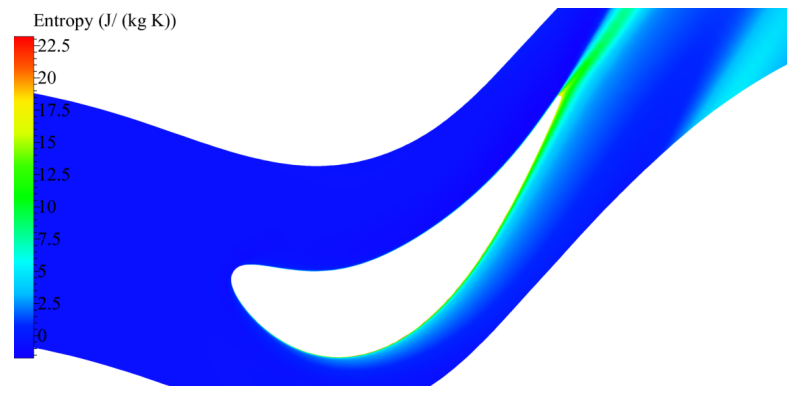

(a) Operating condition A.

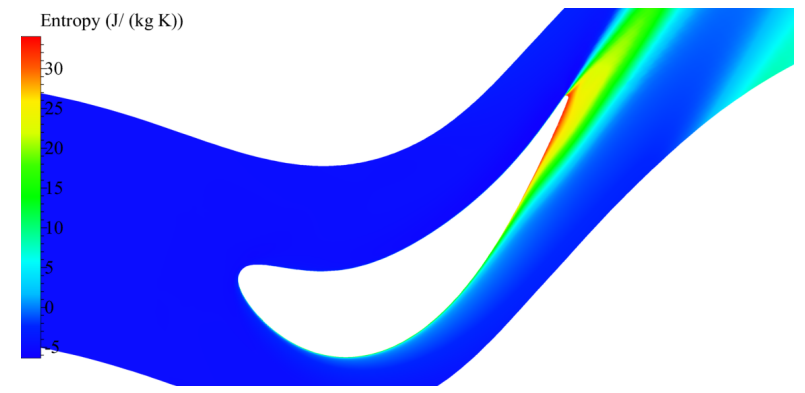

(b) Operating condition B.

Fig. 5 Blade midspan entropy contours from S-EARSM model.

\section{Validation}

Likewise, validation deals with the accuracy of the solution as it relates to experimental data and quantifies the uncertainty of all possible modelling error sources [39]. Unlike verification, there is no method that estimates directly $U_{S M}$ and also $U_{E}$. As an alternative, Stern et al. [14] resorted to the slightly different validation uncertainty defined as

$$
U_{V}^{2}=U_{E}^{2}-U_{S M}^{2}=U_{D}^{2}+U_{S N}^{2}+U_{S P D}^{2},
$$

in which all components have been determined. Hence, validation is achieved if the comparison error is below the noise level, $|E|<U_{V}$. Clearly, Eq. 31 raises a paradox. The more uncertain the numerical solution, the more easier it becomes to validate the CFD code. In this case, the SST model would have more chance to achieve validation despite its lower level of confidence. In the context of a single code, Stern et al. [14] recommended adding a programmatic validation requirement to the procedure to prevent validation at high noise level. However, the authors did not provide any order of magnitude or clear means to determine this parameter. Alternatively, a reasonable suggestion would be breaking the procedure at $U_{S N}>4 U_{D}$ to prevent validation at large numerical uncertainty. 


\begin{tabular}{l|cccccc}
\hline \hline Model & \multicolumn{2}{|c}{ Exp } & \multicolumn{2}{c}{ SST } & \multicolumn{2}{c}{ S-EARSM } \\
Variable & $\bar{D}$ & $U_{D}[\% \bar{D}]$ & $U_{V}\left[\% S_{G 3}^{-}\right]$ & $\bar{E}\left[\% S_{G 3}^{-}\right]$ & $U_{V}\left[\% S_{G 3}^{-}\right]$ & $\bar{E}\left[\% S_{G 3}^{-}\right]$ \\
\hline$p_{A}[\mathrm{~Pa}]$ & $1.302 \times 10^{5}$ & 1.81 & 2.8 & 0.43 & 2.57 & 0.39 \\
$p_{\text {tr }, \text { out }, A}[\mathrm{~Pa}]$ & $1.383 \times 10^{5}$ & 1.82 & 2.65 & 1.12 & 2.6 & 1.02 \\
$\beta_{\text {out }, A}\left[^{\circ}\right]$ & 60.06 & 1.41 & 12.94 & 4.68 & 3.68 & 3.66 \\
$M_{r, \text { out }, A}[-]$ & 0.294 & 1.06 & 5.16 & 2.39 & 3.42 & 1.34 \\
\hline$p_{B}[\mathrm{~Pa}]$ & $1.389 \times 10^{5}$ & 1.81 & 2.63 & 1.39 & 2.56 & 1.35 \\
$p_{\text {tr }, \text { out }, B}[\mathrm{~Pa}]$ & $1.49 \times 10^{5}$ & 1.82 & 2.95 & 1.53 & 2.62 & 1.5 \\
$\beta_{\text {out }, B}\left[{ }^{\circ}\right]$ & 60.53 & 1.41 & 12.45 & 6.75 & 5.32 & 3.97 \\
$M_{r, \text { out }, B}[-]$ & 0.317 & 1.06 & 11.65 & 5.94 & 4.09 & 7.91 \\
\hline \hline
\end{tabular}

Table 7 Validation at rotor exit plane.

In that respect, $\beta$ and $M_{r}$ of the SST model are automatically discarded from the procedure, referring to Tab.7. The S-EARSM model clearly outperforms the SST model at both operating conditions based on the mean value, at the exception of $M_{r, \text { out }, B}$ that can not achieve validation. The high comparison error might be caused by the Merkle preconditioning which did not successfully cover the Aachen turbine low subsonic range $M \in[0.2-0.3]$ as its accuracy has only been confirmed in the range $M \in\left[10^{-6}-0.1\right]$ [13]. In this optic, the setback of $M_{r, \text { out }, B}$ is not absolutely detrimental in the present study since most turbines operate in compressible subsonic range, omitting the need of solver preconditioning. Moreover, it is of interest to assess locally the accuracy of the numerical model basing on the pitch average distribution at the rotor exit. For this purpose, $\beta_{\text {out }}$ distribution is chosen as it serves directly as benchmark for the deviation models.

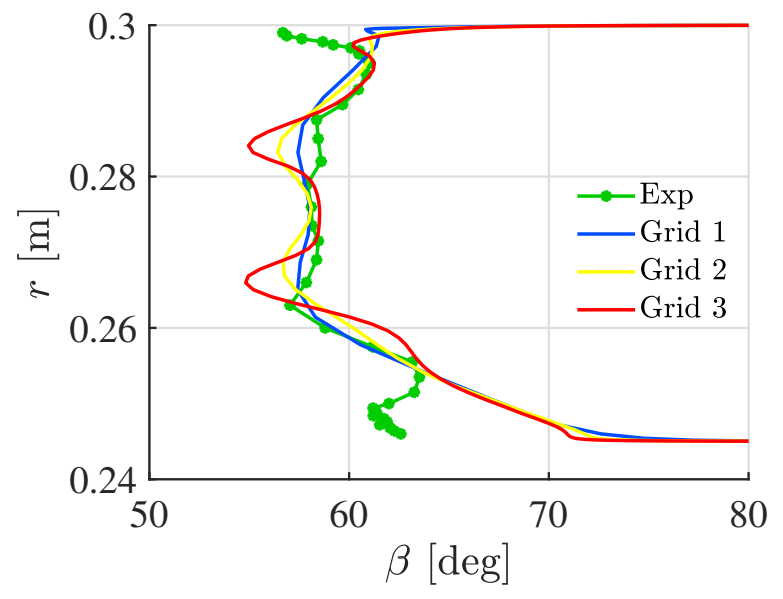

(a) SST model.

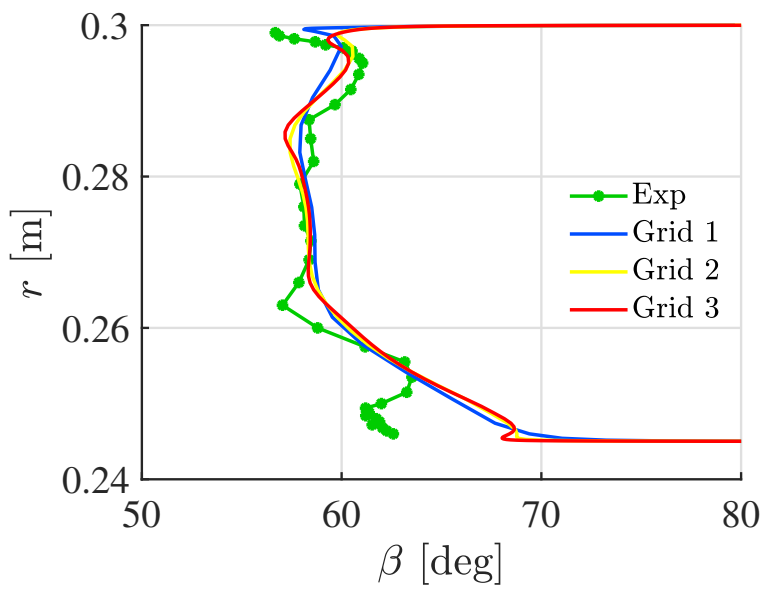

(b) S-EARSM model.

Fig. 6 Pitch average $\beta_{o u t, A}$ distribution.

Near the hub endwall, there is a twisting pattern that originates from the succession of two dominant vortices of opposite skews and is not well captured by the models. Excepting the hub corner flow, the S-EARSM model permits close matching of the results. As for the SST model, two curious peaks spawn from the refined grid near midspan. This anomaly is caused by enhanced motion of overlapping vortices which has no physical basis and thus can only be considered as a pitfall of the SST turbulence modeling. For the sake of completeness, the comparison for the other quantities are gathered in Appx. B. In sum, the S-EARSM model has proven to be superior in terms of accuracy with the $\mathrm{V} \& \mathrm{~V}$ procedure and is therefore retained for the subsequent analysis. 


\section{Results}

The results produced by the outlined methods are gathered and analysed in this section. Recalling briefly the statistical strategy elaborated in Sec. II.C. a descriptive analysis is brought to the compared samples, highlighting essential features. To ascertain the significance of the observed difference between the samples, the inferential T-Test is consecutively conducted in the comparative study. The latter is twofold. The first part demonstrates the superiority of the Aungier model over the KO model in terms of accuracy for axial turbines as intended by its theoretical augmentation. The second part evaluates the suitability of the axial correlations in the context of centrifugal turbine flow by means of an axial benchmark.

\section{Kacker-Okapuu model vs. Aungier model}

Accordingly, the investigation begins with the axial cascades. Two samples generated from the application of the Aungier and KO models are compared. The relative error (involving the CFD solution as defined in Eq. 6) of both loss models are depicted in Fig. 7 and 8 These scatter plots comprise the mean value, the standard deviation and $95 \%$ confidence intervals.

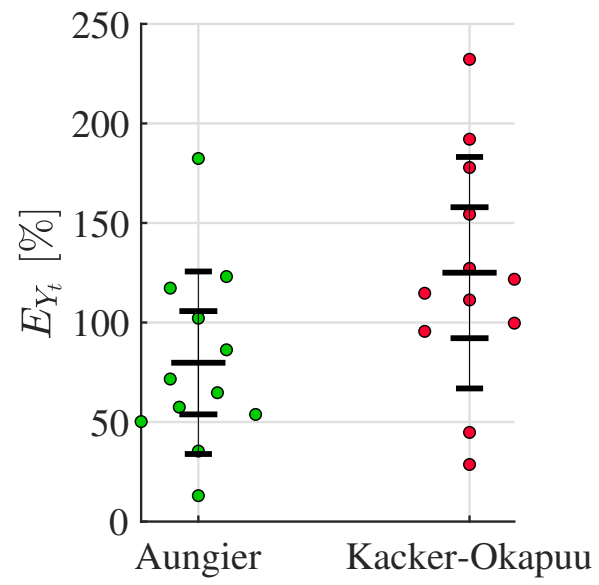

(a) Total pressure loss.

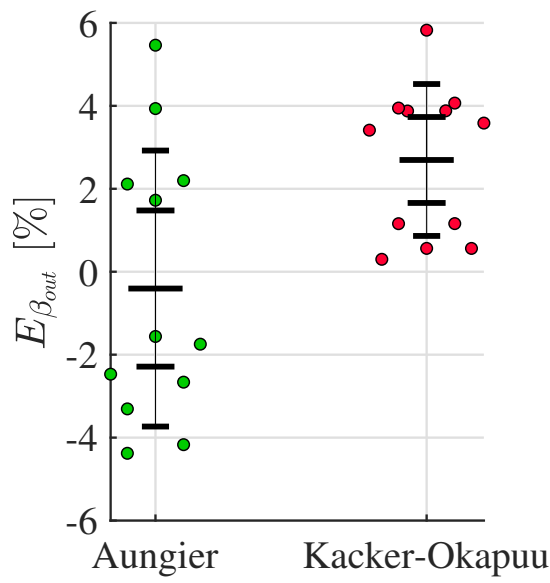

(b) Exit flow angle.

Fig. 7 Error comparison between Aungier and KO models applied to axial stators.

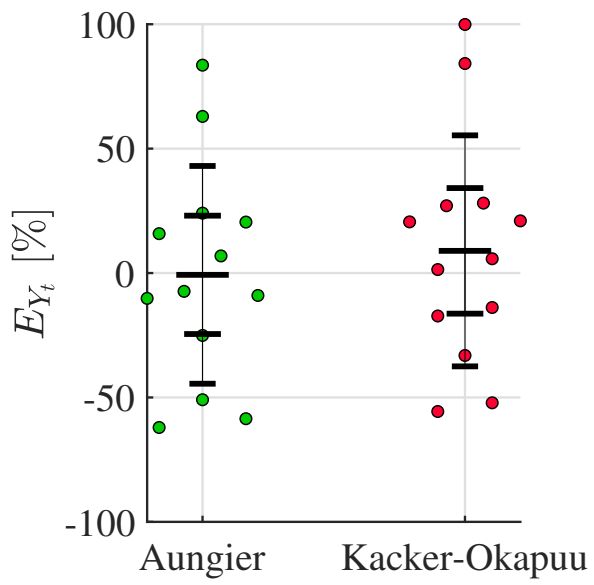

(a) Total pressure loss.

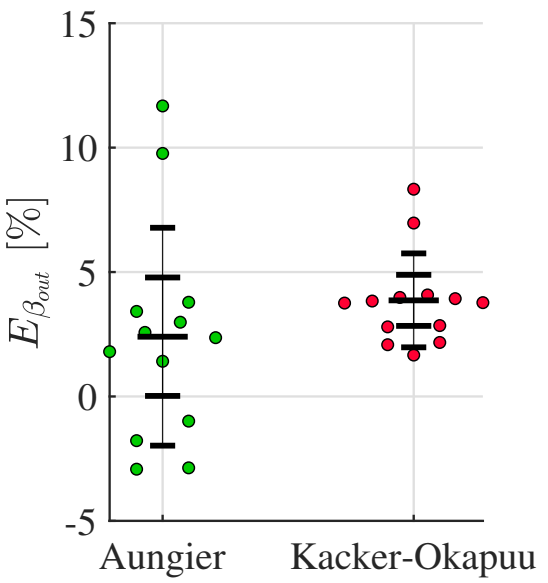

(b) Exit flow angle.

Fig. 8 Error comparison between Aungier and KO models applied to axial rotors. 
Regarding the axial stators, both loss models yield drastic surplus of total loss with respect to that of the simulation. Quantitatively, the Aungier and KO models overpredict by approximately $80 \%$ and $125 \%$ in average respectively. As for those of the axial rotors, an ideal behavior is noted with the mean values in the vicinity of $0 \%$ and $10 \%$ respectively. By evidence, the Aungier model exhibits lower mean errors. The confidence interval covering $\pm 30 \%$ range remains constant regardless of the cascade configuration and loss model. This is expected since the Aungier and KO models share fundamentally the same formulation. From this single observation, the error's order of magnitude uncovered in both cascades agrees with that of Benner et al. [35]. The latter stipulates that the KO model is actually calibrated to rotating rigs which comprise more loss generating mechanisms than their static counterpart. Given the results, this also applies for the Aungier model.

On the opposite, there are no large scale error nor differences for the outlet flow angle. Nonetheless, most points of the stator fall outside of the one standard deviation interval, giving notably a mean estimated with extreme values, whereas those of the rotor are more evenly distributed. The AM model systematically exhibits slightly higher mean error but produces consistent results with narrower standard deviation by contrast to the Aungier model. This can be explained by their different nature as shown in Eq. 13 and 17. The AM model predicts directly the outlet flow angle whereas the Aungier model requires the blade outlet metal angle beforehand in order to deduce the outlet flow angle.

Yet, the given descriptive analysis of the samples is limited as it can not provide a generalization of the observed patterns. Instead, the latter is acquired with the inferential T-Test as complementary method to the analysis. Note that the T-Test can only prove whether or not there is an equivalence between the considered samples, corresponding mathematically to a "=" or " $\neq "$ condition test. In this context, imbalance condition corresponding to "<" or "> has to be incorporated to ascertain the accuracy superiority of a model. For this purpose, the observed patterns and error scale are assumed to be preserved in any experiment reproduction.

The results for both cascades are gathered in Tab. 8 in which $\mathrm{P}$ correspond to the probability of occurrence of the condition $m_{1} \neq m_{2}$.

\begin{tabular}{l|ccc}
\hline \hline Parameters & T [-] & P [\%] & $m_{1} \neq m_{2}$ \\
\hline Stator $E_{Y_{t}}$ & 6.81 & 99.99 & Yes \\
Stator $E_{\beta_{\text {out }}}$ & 3.17 & 99.11 & Yes \\
Rotor $E_{Y_{t}}$ & 5.08 & 99.97 & Yes \\
Rotor $E_{\beta_{\text {out }}}$ & 1.357 & 80.03 & No \\
\hline \hline
\end{tabular}

Table 8 Results of the T-Test for KO and Aungier models applied to axial cascades.

For the outlet flow angle, the result is one-sided, aborting prematurely the analysis. In that respect, no conclusion can be drawn on the accuracy of the deviation models using the inferential method. Still, it is reasonable to suggest that the AM model should be preferred as it involves less uncertainty according to the descriptive analysis.

Regarding the loss, the difference is proven to be significant statistically for both cascade configurations. Combining with the previous observation, it is concluded that the Aungier model is superior to the KO model in terms of accuracy.

\section{Axial cascades vs. Centrifugal cascades}

Before proceeding to the analysis of the results, it is worthwhile to outline two main aspects inherent to the centrifugal flow that alter the quantities taken at the blade outlet and thereafter the axial correlations. The first is the increasing flow path area that naturally diffuses the radial outward flow. The second concerns rotation that prompts the additional fictive centrifugal and Coriolis forces. Their superposition results in a significant streamwise increase of static pressure that in return restrains inter-blade flow acceleration. Hence, lower velocity field and thicker boundary layer buildup as shown by the contour in Fig. 9 contributing to outlet Mach number reduction and loss increase are foreseen. 


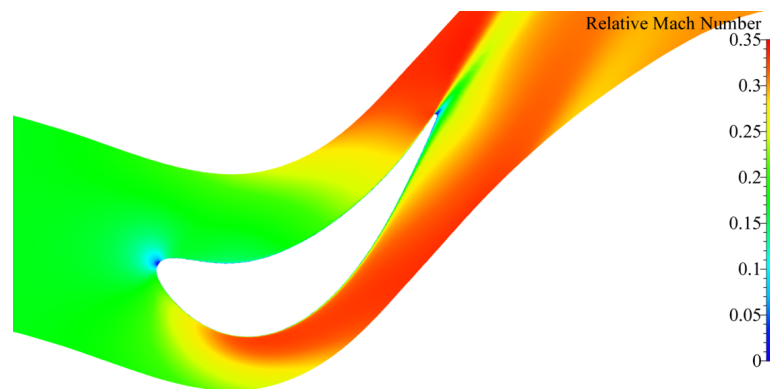

(a) Axial.

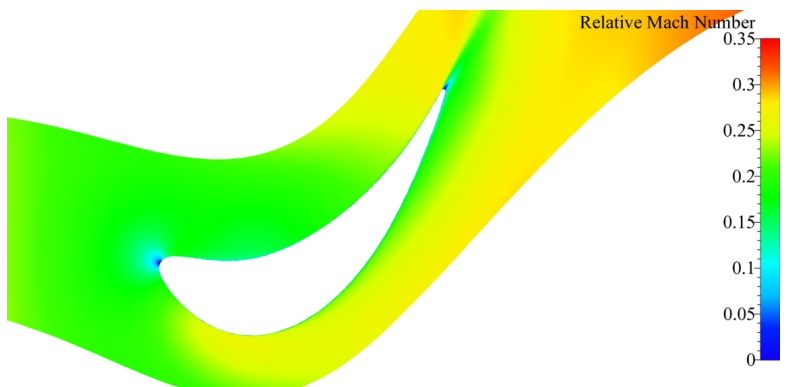

(b) Centrifugal.

Fig. 9 Blade-to-blade relative Mach number contour of R1 [15].

Moreover basing on the results of the previous analysis over the axial cascades, it is notably inferred that the loss models were calibrated to the rotors only. Thus, the comparative study is perpetuated with rotors in order to obtain a reliable axial benchmark. In the same vein, two samples representing the axial and centrifugal rotors are compared through the application of the Aungier loss and AM deviation models. The relative loss error and corresponding loss quantities are still depicted with scatter plots Fig. 10.

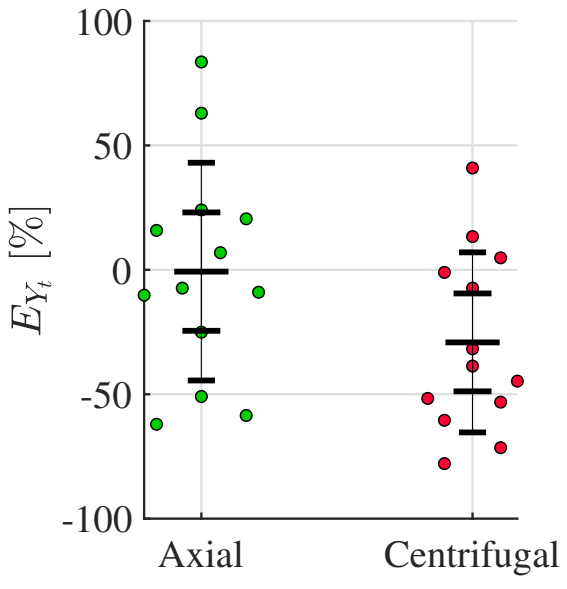

(a) Loss error.

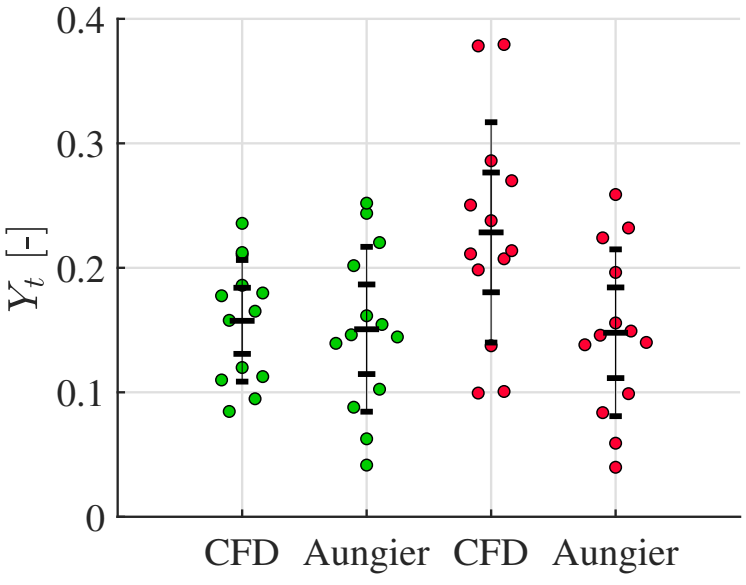

(b) Corresponding total loss.

Fig. 10 Loss comparison between CFD and Aungier model applied to axial and centrifugal rotors.

The evident error and loss disparities point out the fact that there exists either an underestimation from the Aungier model or an overprediction of the CFD simulations. Recalling the centrifugal flow features cited earlier and the non-optimal configuration acquired through conformal mapping, the loss increase of the centrifugal cascade given by CFD is well-founded. Thus, the remaining option indicates that the issue lies in the Aungier model. In fact, it is seen that the losses of the centrifugal sample nearly match those of the axial sample. Considering their geometrical similarity, this insensitivity implies that the operational parameters actually have little influence over the loss generation regardless of the configuration.

Likewise, the relative angle error and corresponding flow angles are shown in Fig. 11. Remarkably, the errors associated to the centrifugal cascades exhibit a better behavior with lower mean and narrower standard deviation width and these are backed by the flow angles delivered by the AM model that are actually closer to the CFD solutions. This contrast with the axial cascade originates from the specific formulations in Eq. 13 and 14 which involve an arbitrary linear interpolation with the outlet Mach number in the range $M_{r, \text { out }} \in[0.5-1]$. Since most axial rotors operate in high subsonic condition, these are more exposed to the additional errors brought by the arbitrary interpolation contrary to the centrifugal rotors which operate in considerably lower Mach number range. Furthermore, the CFD solutions of both cascades are similar on average, implying that the centrifugal flow does not severely alter the outlet flow deviation. 


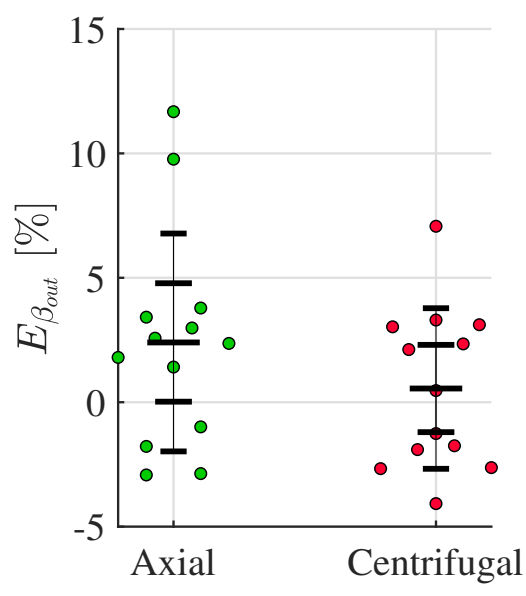

(a) Outlet flow angle error.

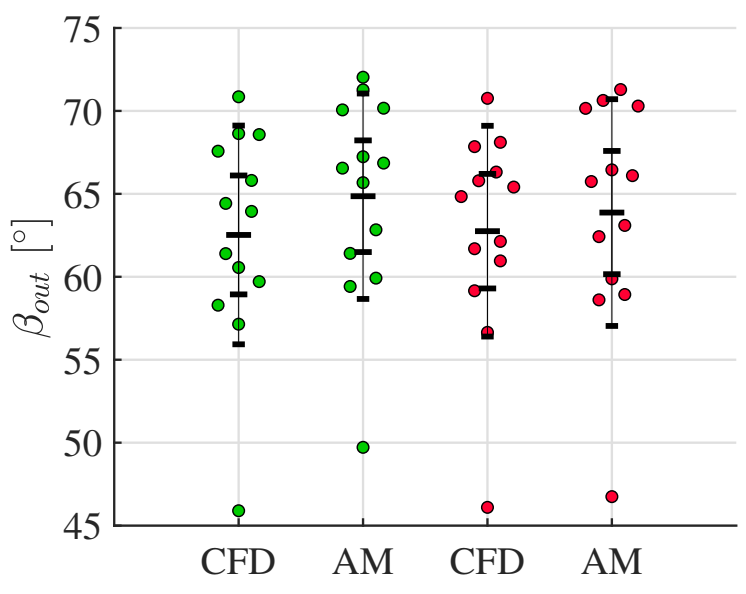

(b) Corresponding outlet flow angle.

Fig. 11 Flow angle comparison between CFD and AM model applied to axial and centrifugal rotors.

Again, the observations have to be ascertained with the T-Test. Assuming reproducibility of the samples patterns, the results are shown in Tab.9

\begin{tabular}{l|ccc}
\hline \hline Parameters & $\mathrm{T}[-]$ & $\mathrm{P}[\%]$ & $m_{1} \neq m_{2}$ \\
\hline Rotor $Y_{t, \text { model }}$ & 0.94 & 63.82 & No \\
Rotor $\beta_{\text {out }, \text { model }}$ & 3.06 & 99.02 & Yes \\
Rotor $E_{Y_{t}}$ & 4.37 & 99.9 & Yes \\
Rotor $E_{\beta_{\text {out }}}$ & 2.65 & 97.88 & Yes \\
\hline \hline
\end{tabular}

Table 9 Results of the T-Test applied to axial and centrifugal rotors.

There are no significant difference between the losses of both twin cascades as delivered by the Aungier model, confirming that the operational parameters are of least impact. Moreover, large errors or inconsistencies would remain present if the Aungier model is still applied to centrifugal cascade according to the relative error hypothesis test. Hence, it is concluded that axial correlations built upon axial turbine flow measurements are exclusive to axial turbines and should not be extended to the performance prediction of centrifugal turbines.

By contrast, the results of the flow angle and its relative error hypothesis tests ascertain the suitability of the AM deviation model in centrifugal turbine flows. By the same occasion, it is also advanced that the Mach number interpolation in the high subsonic range effectively is responsible for the additional error observed in the axial cascades.

\section{Conclusion}

A comparative study has been conducted on twin axial and centrifugal cascades to evaluate the appropriateness of axial correlations in centrifugal turbine performance prediction. The descriptive analysis complemented with inferential hypothesis tests reliably advances two conclusions :

1) The axial loss models represented by the $\mathrm{KO}$ and Aungier models in this study is unable to capture the aerodynamics of the centrifugal turbine flow and thus should remain exclusive to the axial turbines.

2) The axial deviation models which have a direct reliance on the blade geometrical disposition are suited for use in centrifugal turbines. 
Although the axial loss models have proven to be inadequate, it could still provide the basis for the elaboration of a proper centrifugal turbine loss model. For this end, a more in-depth sensitivity analysis on each loss component should be conducted in order to assess the actual impact of the operational and geometrical parameters and identify the functions that could undergo calibration to the centrifugal flow.

\section{Nomenclature}

\begin{tabular}{|c|c|c|}
\hline$r$ & $=$ & radius $[\mathrm{m}]$ \\
\hline$\beta$ & $=$ & gas angle $\left[{ }^{\circ}\right]$ \\
\hline$p$ & $=$ & static pressure $[\mathrm{Pa}]$ \\
\hline$T$ & $=$ & static temperature $[\mathrm{K}]$ \\
\hline$\Omega$ & $=$ & rotation speed [RPM] \\
\hline$\dot{m}$ & $=$ & mass flow $[\mathrm{kg} / \mathrm{s}]$ \\
\hline$e$ & $=$ & back curvature radius $[\mathrm{m}]$ \\
\hline$k$ & $=$ & specific heat ratio $[-]$ \\
\hline$M$ & $=$ & Mach number [-] \\
\hline$\psi$ & $=$ & blade loading [-] \\
\hline$\phi$ & $=$ & flow coefficient [-] \\
\hline$\eta$ & $=$ & efficiency [-] \\
\hline$m_{1}, m_{2}$ & $=$ & samples mean $[-]$ \\
\hline$N_{1}, N_{2}$ & $=$ & samples size $[-]$ \\
\hline$v$ & $=$ & kinematic viscosity $\left[\mathrm{m}^{2} / \mathrm{s}\right]$ \\
\hline$K_{R e}$ & $=$ & Reynolds number correction factor [-] \\
\hline$K_{p}$ & $=$ & compressibility correction factor [-] \\
\hline$K_{\text {inc }}$ & $=$ & incidence correction factor [-] \\
\hline$Z$ & $=$ & Ainley loading parameter [-] \\
\hline E & $=$ & solution error $[-]$ \\
\hline$S$ & $=$ & solution [-] \\
\hline$r_{G}$ & $=$ & grid refinement ratio [-] \\
\hline$\delta_{R E}$ & $=$ & numerical error of RE [-] \\
\hline$C_{G}$ & $=$ & correction factor of RE [-] \\
\hline
\end{tabular}

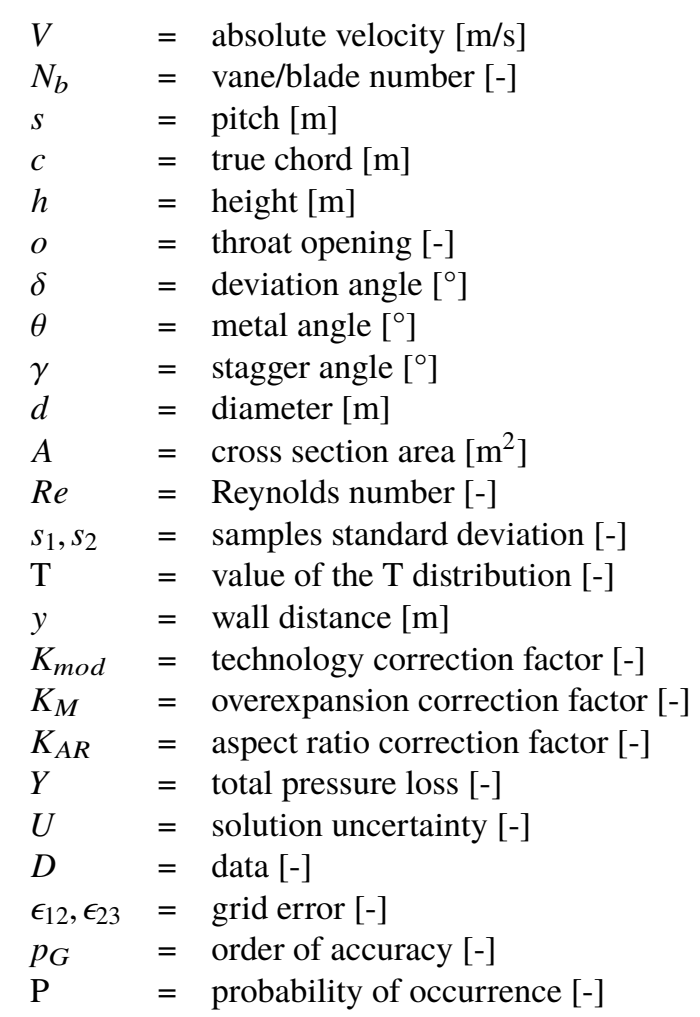

\section{Subscript}

$\begin{array}{ll}\text { in } & =\text { inlet } \\ h u b & =\text { hub } \\ t & =\text { total } \\ t e & =\text { trailing edge } \\ A M & =\text { Ainley-Mathieson } \\ M & =\text { modelling } \\ G & =\text { grid } \\ D & =\text { data }\end{array}$

\section{Acronym}

$\begin{array}{ll}\text { out } & =\text { outlet } \\ r & =\text { relative } \\ p & =\text { profile } \\ s h & =\text { shock waves } \\ D C & =\text { Dunham-Came } \\ P D & =\text { previous data } \\ T & =\text { time } \\ S & =\text { simulation }\end{array}$

$$
\begin{aligned}
\text { tip } & =\text { tip } \\
o & =\text { throat } \\
s & =\text { secondary } \\
H & =\text { hydraulic } \\
N & =\text { numerical } \\
I & =\text { iterative } \\
E & =\text { error } \\
V & =\text { validation }
\end{aligned}
$$

$\begin{array}{ll}\text { AM } & =\text { Ainley-Mathieson } \\ \text { KO } & =\text { Kacker-Okapuu } \\ \text { CFD } & =\text { Computational Fluid Dynamics } \\ \text { STVD } & \text { Symmetric Total Variational Diminishing } \\ \text { FAS } & =\text { Full Approximation Storage } \\ \text { WJ } & =\text { Wallin-Johansson } \\ \text { BSL } & =\text { Baseline } \\ \text { VKI } & =\text { Von Karman Institute }\end{array}$

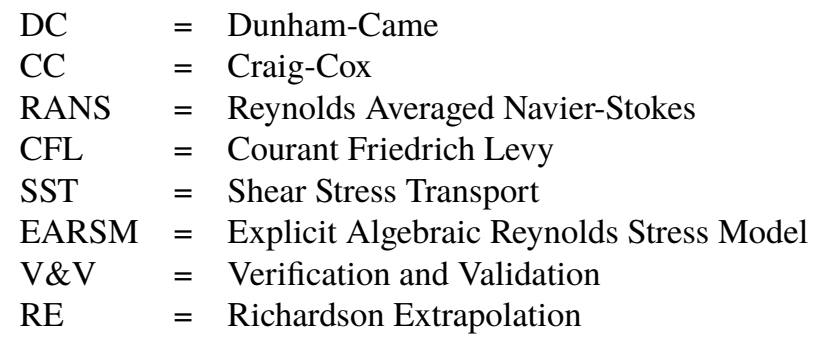




\section{References}

[1] Grönman, A., Uusitalo, A., and Backman, J., "Loss Generation in Radial Outflow Steam Turbine Cascades," ETC Paper ETC2017-039, 2017.

[2] Spadacini, C., Centemeri, L., Xodo, L. G., Astolfi, M., Romano, M. C., and Macchi, E., “A New Configuration for Organic Rankine Cycle Power Systems,” International Seminar on ORC Power Systems, 2011.

[3] Rodríguez, L., Pereiras, B., Fernandez-Oro, J., and Castro, F., "Viability of Unidirectional Radial Turbines for Twin-Turbine Configuration of OWC Wave Energy Converters," Elsevier Ocean Engineering, Vol. 154, 2018.

[4] Stodola, A., and Lowenstein, L. C., Steam and Gas Turbines, $1^{\text {st }}$ ed., McGraw-Hill, 1927.

[5] Pini, M., Persico, G., Casati, E., and Dossena, V., "Preliminary Design of a Centrifugal Turbine for Organic Rankine Cycle Applications," ASME Journal of Engineering for Gas Turbines and Power, Vol. 135, 2013.

[6] Luo, D., Tan, X., and Huang, D., "Design and Performance Analysis of Three Stage Centrifugal Turbine," Elsevier Applied Thermal Engineering, Vol. 138, 2018.

[7] Klonowicz, P., Heberle, F., Preißinger, M., and Brüggemann, D., "Significance of Loss Correlations in Performance Prediction of Small Scale, Highly Loaded Turbine Stages Working in Organic Rankine Cycles,” Elsevier Energy, Vol. 72 , 2014.

[8] Ainley, D. G., and Mathieson, G. C. R., "A Method of Performance Estimation for Axial Flow Turbines," British ARC, R\&M $2974,1951$.

[9] Dunham, J., "A Review of Cascade Data on Secondary Losses in Turbines," SAGE Journal of Mechanical Engineering Science, Vol. 12, 1970.

[10] Kacker, S. C., and Okapuu, U., "A Mean Line Prediction Method for Axial Flow Turbine Efficiency," ASME Journal of Engineering for Power, Vol. 104, 1982.

[11] Persico, G., Pini, M., Dossena, V., and Gaetani, P., "Aerodynamic Design and Analysis of Centrifugal Turbine Cascades," ASME Paper GT2013-95770, 2013.

[12] Aungier, R. H., Turbine Aerodynamics : Axial-Flow and Radial-Inflow Turbine Design and Analysis, $1^{\text {st }}$ ed., ASME Press, 2006.

[13] FINE/Turbo 14.2 Theory Guide, 2020.

[14] Stern, F., Wilson, R. V., Coleman, H. W., and Paterson, E. G., "Comprehensive Approach to Verification and Validation of CFD Simulations-Part 1: Methodology and Procedures,” ASME Journal of Fluids Engineering, Vol. 123, 2001.

[15] Walraevens, R. E., and Gallus, H. E., “Testcase 6 : 1.5 Stage Axial Flow Turbine,” ERCOFTAC, 1997.

[16] Baskharone, E. A., Principles of Turbomachinery in Air-Breathing Engines, $1^{\text {st }}$ ed., Cambridge University Press, 2006.

[17] Hirsch, K., and Moghadam, R., "Validation of One Dimensional Loss Models for Axial Gas Turbines," Master's thesis, Lund University, 2016.

[18] Behr, T., "Control of Rotor Tip Leakage and Secondary Flow by Casing Air Injection in Unshrouded Axial Turbines," Ph.D. thesis, ETH Zürich, 2007.

[19] Dring, R. P., Fair, M. F., Joslyn, H. D., Power, G. D., and Verdon, J. M., “The Effect of Inlet Turbulence and Rotor/Stator Interactions on the Aerodynamics and Heat Transfer of a Large Scale Rotating Rig Turbine Model, Vol. I - Final Report," NASA Contractor Report 4079, 1987.

[20] Stabe, R. G., Whitney, W. J., and Moffit, T. P., "Performance of a High-Work Low Aspect Ratio Turbine Tested With a Realistic Radial Temperature Profile," NASA TM-83655, 1984.

[21] Timko, L. P., "Energy Efficient Engine High Pressure Turbine Component Test Performance Report,” NASA-CR-168289, 1984.

[22] Cherry, D. G., Gay, C. H., and Lenahan, D. T., "Energy Efficient Engine Low Pressure Turbine Test Hardware Detailed Design Report," NASA-CR-167956, 1984.

[23] Kotzing, P., and Evers, B., "Testcase E/TU-4 4-Stage Low Speed Turbine, Test Cases for Computation of Internal Flows in Aero Engine Components," AGARD-AR-257, 1990. 
[24] NREC, C., "Disclosed testcases from commercial software AxCent database,", 2020.

[25] Persson, M., "Highly Loaded HPT Blading in KTH Test Turbine," Master’s thesis, Lund University, 2015.

[26] Leach, K., Thulin, R. D., and Howe, D. C., "Energy Efficient Engine: Turbine Intermediate Case and Low-Pressure Turbine Component Test Hardware Detailed Design Report," NASA-CR-167973, 1984.

[27] Mendenhall, W., Beaver, R. J., and Beaver, B. M., Introduction to Probability and Statistics, 14 ${ }^{\text {th }}$ ed., Brooks Cole, 2013.

[28] Morgan, C. J., "Use of Proper Statistical Techniques for Research Studies with Small Samples," APS American Journal of Physiology-Lung Cellular and Molecular Physiology, Vol. 313, 2017.

[29] Baturin, O., Kolmakova, D., Gorshkov, A., and Popov, G., "Selection of Models to Assess the Profile Losses in Blade Rows Using the Methods of Mathematical Statistics," ASME Paper GTINDIA2015-1245, 2015.

[30] Davidson, P. A., Turbulence : An Introduction for Scientists and Engineers, $2^{\text {nd }}$ ed., Oxford University Press, 2015.

[31] Dunham, J., and Came, P. M., "Improvements to the Ainley-Mathieson Method of Turbine Performance Prediction," ASME Journal of Engineering for Power, Vol. 92, 1970.

[32] Lozza, G., "A Comparison between the Craig-Cox and the Kacker-Okapuu Methods of Turbine Performance Prediction," Meccanica, Vol. 17, 1982.

[33] Wei, N., "Significance of Loss Models in Aerothermodynamic Simulation for Axial Turbines," Ph.D. thesis, Kungliga Tekniska Högskolan, 2000.

[34] Benini, E., Boscolo, G., and Garavello, A., "Assessment of Loss Correlations for Performance Prediction of Low Reaction Gas Turbine Stages," ASME Paper IMECE2008-69085, 2008.

[35] Benner, M. W., Sjolander, S. A., and Moustapha, S. H., "An Empirical Prediction Method For Secondary Losses In Turbines—Part II: A New Secondary Loss Correlation,” ASME Journal of Turbomachinery, Vol. 128, 2006.

[36] Craig, H. R. M., and Cox, H. J. A., "Performance Estimation of Axial Flow Turbines," SAGE Proceedings, Institution of Mechanical Engineers, Vol. 185, 1970.

[37] Da Lio, L., Manente, G., and Lazaretto, A., "New Efficiency Charts for the Optimum Design of Axial Flow Turbines for Organic Rankine Cycles," Elsevier Energy, Vol. 77, 2014.

[38] Denton, J. D., "Some Limitations of Turbomachinery CFD,” ASME Paper GT2010-22540, 2010.

[39] Oberkampf, W. L., and Blottner, F. G., "Issues in Computational Fluid Dynamics Code Verification and Validation," AIAA Journal, Vol. 36, 1998.

[40] Stern, F., Wilson, R. V., and Shao, J., "Quantitative V\&V of CFD Simulations and Certication of CFD Codes,” Wiley International Journal for Numerical Methods in Fluids, Vol. 50, 2006.

[41] Walraevens, R. E., Gallus, H. E., Jung, A. R., Mayer, J. F., and Stetter, H., "Experimental and Computational Study of the Unsteady Flow in a 1.5 Stage Axial Turbine with Emphasis on the Secondary Flow in the Second Stator," ASME Paper 98-GT-254, 1998.

[42] Rizzi, A., Eliasson, P., Lindblad, I., Hirsch, C., Lacor, C., and Haeuser, J., "The Engineering of Multiblock/Multigrid Software for Navire Stokes Flows on Structured Meshes," Elsevier Computers Fluids, Vol. 22, 1993.

[43] Hirsch, C., Fundamentals of Computational Fluid Dynamics, $2^{\text {nd }}$ ed., Numerical Computation of Internal and External Flows, Vol. 1, Butterworth-Heinemann, 2007.

[44] Jameson, A., Schmidt, W., and Turkel, E., "Numerical Solutions of the Euler Equations by Finite Volume Methods Using Runge-Kutta Time-Stepping Schemes," AIAA Paper 81-1259, 1981.

[45] Yee, H. C., "Construction of Explicit and Implicit Symmetric TVD Schemes and their Applications," Elsevier Journal of Computational Physics, Vol. 68, 1987.

[46] Radespiel, R., Rossow, C., and Swanson, R. C., "Efficient Cell-Vertex Multigrid Scheme for the Three-Dimensional Navier-Stokes Equations," AIAA Journal, Vol. 28, 1990. 
[47] Choi, Y. H., and Merkle, C. L., "The Application of Preconditioning in Viscous Flows," Elsevier Journal of Computational Physics, Vol. 105, 1993.

[48] Menter, F. R., “Two-Equation Eddy-Viscosity Turbulence Models for Engineering Applications,” AIAA Journal, Vol. 32 , 1994.

[49] Menter, F. R., “Zonal Two Equation $k-\omega$, Turbulence Models for Aerodynamic Flows,” AIAA Paper 93-2906, 1993.

[50] Wilcox, D. C., "Reassessment of the Scale-Determining Equation for Advanced Turbulence Models," AIAA Journal, Vol. 26, 1988.

[51] Launder, B. E., and Sharma, B. I., "Application of the Energy Dissipation Model of Turbulence to the Calculation of Flow near a Spinning Disk," Letters in Heat and Mass Transfer, Vol. 1, 1974.

[52] Bradshaw, P., "Turbulence Modeling with Application to Turbomachinery," Elsevier Progress in Aerospace Sciences, Vol. 32, 1996.

[53] Lakshminarayana, B., Fluid Dynamics and Heat Transfer of Turbomachinery, $1^{\text {st }}$ ed., Vol. 1, Wiley, 1996.

[54] Menter, F. R., Garbaruk, A. V., and Egorov, Y., "Explicit Algebraic Reynolds Stress Models for Anisotropic Wall-Bounded Flows," EDP Progress in Flight Physics, Vol. 3, 2012.

[55] Wallin, S., and Johansson, A. V., "An Explicit Algebraic Reynolds Stress Model for Incompressible and Compressible Turbulent Flows," Cambridge Journal of Fluid Mechanics, Vol. 403, 2000.

[56] Mehdizadeh, O. Z., Temmerman, L., Tartinville, B., and Hirsch, C., "Applications of EARSM Turbulence Models to Internal Flows," ASME Paper GT2012-68886, 2012.

[57] Wickerath, B., and Niehuis, R., "A Study of Nonlinear Eddy Viscosity Models in a Flow Solver for Turbomachinery," ICAS 25th meeting paper, 2006.

[58] Walker, G. J., “Transitional Flow on Axial Turbomachine Blading,” AIAA Journal, Vol. 27, 1989.

[59] Rumsey, C. L., "Apparent Transition Behavior of Widely-Used Turbulence Models," Elsevier International Journal of Heat and Fluid Flow, Vol. 28, 2007.

[60] Dick, E., and Kubacki, S., "Transition Models for Turbomachinery Boundary Layer Flows: A Review," MDPI International Journal of Turbomachinery Propulsion Power, Vol. 2, 2017.

[61] Kelterer, M. E., Pecnik, R., and Sanz, W., "Computation of Laminar-Turbulent Transition in Turbomachinery Using the Correlation Based $\gamma-\operatorname{Re}_{\theta}$ Transition Model,” ASME Paper GT2010-22207, 2010.

[62] Langtry, R. B., and Menter, F. R., "Correlation-Based Transition Modeling for Unstructured Parallelized Computational Fluid Dynamics Codes," AIAA Journal, Vol. 47, 2009.

[63] Persico, G., Pini, M., Dossena, V., and Gaetani, P., “Aerodynamics of Centrifugal Turbine Cascades," ASME Journal of Engineering for Gas Turbines and Power, Vol. 137, 2015.

[64] Simonsen, C. D., and Stern, F., "Verification and Validation of RANS Maneuvering Simulation of Esso Osaka: Effects of Drift and Rudder Angle on Forces and Moments," Elsevier Computer \& Fluids, Vol. 32, 2003.

[65] Baker, T. J., "Mesh Generation : Art or Science?” Elsevier Progress in Aerospace Sciences, Vol. 41, 2005.

[66] Turgut, O. H., and Camci, C., "Factors Influencing Computational Predictability of Aerodynamic Losses in a Turbine Nozzle Guide Vane Flow," ASME Journal of Fluids Engineering, Vol. 138, 2016.

[67] Hirsch, C., and Tartinville, B., "Reynolds-Averaged Navier-Stokes Modelling for Industrial Applications and some Challenging Issues," Taylor \& Francis International Journal of Computational Fluid Dynamics, Vol. 23, 2009.

[68] Volmar, T. M., Brouillet, B., Gallus, H. E., and Benetschik, H., "Time Accurate 3D Navier Stokes Analysis of a 1.5 Stage Axial Turbine," AIAA Paper 98-3247, 1998.

[69] Utz, C., "Experimentelle Untersuchung der Strömungsverluste in einer mehrstufigen Axialturbine,” Ph.D. thesis, ETH Zürich, 1972.

[70] Aubé, M., and Hirsch, C., "Numerical Investigation of a 1.5 Axial Turbine at Quasi-Steady and Fully Unsteady Conditions," ASME Paper 2001-GT-0309, 2001.

[71] Roy, C. J., "Review of Code and Solution Verification Procedures for Computational Simulation," Elsevier Journal of Computational Physics, Vol. 205, 2005. 


\section{A. List of Turbines Geometry}

\begin{tabular}{l|cccccc}
\hline \hline Parameters & $N_{b}[-]$ & $h / c[-]$ & $c[\mathrm{~mm}]$ & $\theta_{\text {in }}\left[^{\circ}\right]$ & $\theta_{\text {out }}\left[^{\circ}\right]$ & $\gamma\left[{ }^{\circ}\right]$ \\
\hline $1^{\text {st }}$ Stage Stator S1 [15] & 36 & 0.887 & 62 & 0 & -70 & -44.5 \\
$1^{\text {st }}$ Stage Stator S2 [17] & 42 & 0.988 & 34.41 & 33.46 & -74.65 & -46 \\
$1^{\text {st }}$ Stage Stator S3 [18] & 36 & 0.87 & 80.88 & 0 & -72 & -50.2 \\
$1^{\text {st }}$ Stage Stator S4 [19] & 22 & 0.663 & 229.35 & 0 & -68.58 & -48.82 \\
$1^{\text {st }}$ Stage Stator S5 [20] & 26 & 0.509 & 70 & 0 & -75 & -59.55 \\
$1^{\text {st }}$ Stage Stator S6 [21] & 46 & 0.66 & 60.6 & 0 & -74.2 & -56.12 \\
$2^{\text {nd }}$ Stage Stator S7 [21] & 48 & 1.047 & 65.2 & 21.8 & -69 & -41.21 \\
$1^{\text {st }}$ Stage Stator S8 [22] & 72 & 1.069 & 44.7 & 0 & -60 & -37.6 \\
$2^{\text {nd }}$ Stage Stator S9 [22] & 102 & 2.587 & 28.6 & 53.95 & -60.8 & -22.90 \\
$1^{\text {st }}$ Stage Stator S10 [23] & 29 & 1.057 & 56.3 & 0 & -68.32 & -34.61 \\
$1^{\text {st }}$ Stage Stator S11 [24] & 57 & 2.23 & 27.44 & 0 & -54.88 & -38.25 \\
$1^{\text {st }}$ Stage Stator S12 [24] & 93 & 1.44 & 27.15 & 0 & -60.53 & -42.79 \\
\hline $1^{\text {st }}$ Stage Rotor R1 [15] & 41 & 0.917 & 60 & -40.7 & 61.2 & 28 \\
$1^{\text {st }}$ Stage Rotor R2 [17] & 58 & 1.303 & 26.08 & -58.3 & 70.37 & 23.05 \\
Rotor R3 [25] & 60 & 1.122 & 23.6 & -29 & 71.39 & 42.46 \\
Rotor R4 [25] & 50 & 1.031 & 25.7 & -33 & 71.1 & 47.46 \\
$1^{\text {st }}$ Stage Rotor R5 [18] & 54 & 1.17 & 59.72 & -52.4 & 66.6 & 35.5 \\
$1^{\text {st } S t a g e ~ R o t o r ~ R 6 ~[19] ~}$ & 28 & 0.803 & 189.3 & -47.81 & 64.03 & 31.74 \\
$1^{\text {st }}$ Stage Rotor R7 [20] & 48 & 0.913 & 39 & -45 & 67.9 & 32.14 \\
$1^{\text {st }}$ Stage Rotor R8 [21] & 76 & 1.19 & 35.8 & -43.2 & 66.9 & 36.7 \\
$2^{\text {nd }}$ Stage Rotor R9 [21] & 70 & 1.81 & 38.5 & -17 & 59.8 & 37.04 \\
$1^{\text {st }}$ Stage Rotor R10 [22] & 120 & 3.28 & 20.3 & -47.9 & 61.78 & 24.58 \\
$2^{\text {nd }}$ Stage Rotor R11 [22] & 122 & 4.13 & 20.7 & -49.3 & 67.88 & 26.65 \\
$1^{\text {st }}$ Stage Rotor R12 [26] & 120 & 2.82 & 29.4 & -53.6 & 68.66 & 26.4 \\
$1^{\text {st }}$ Stage Rotor R13 [24] & 69 & 2.69 & 22.7 & -21.09 & 52.72 & 22.06 \\
\hline & & & & & & \\
\hline
\end{tabular}

Table 10 Axial stator and rotor geometry parameters. 


\section{B. Aachen turbine rotor exit field}

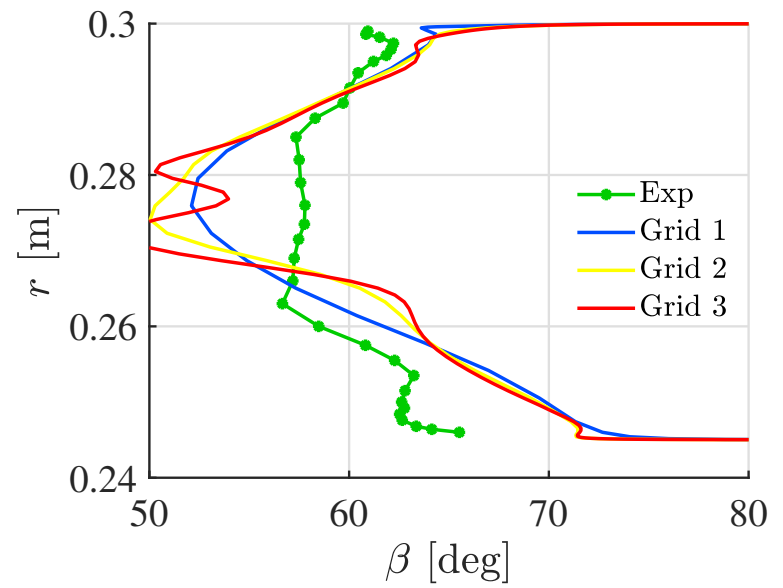

(a) SST model.

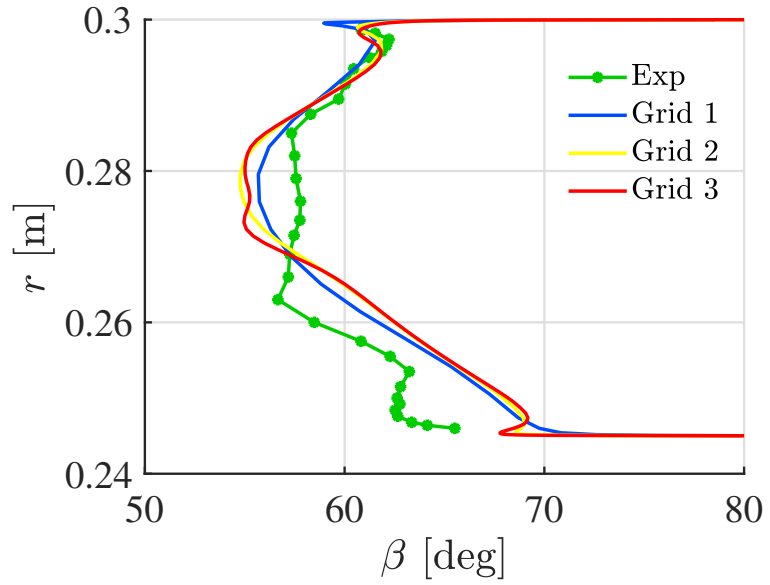

(b) S-EARSM model.

Fig. 12 Pitch average $\beta_{o u t, B}$ distribution.

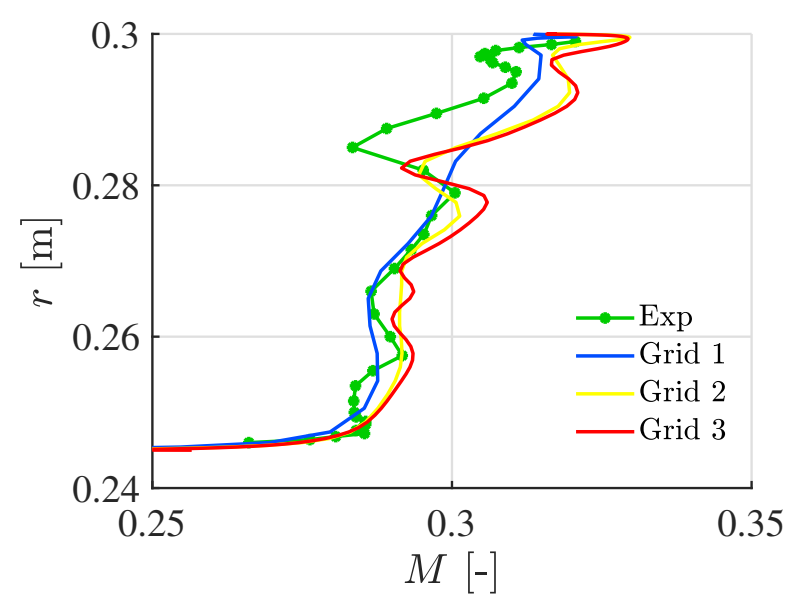

(a) SST model.

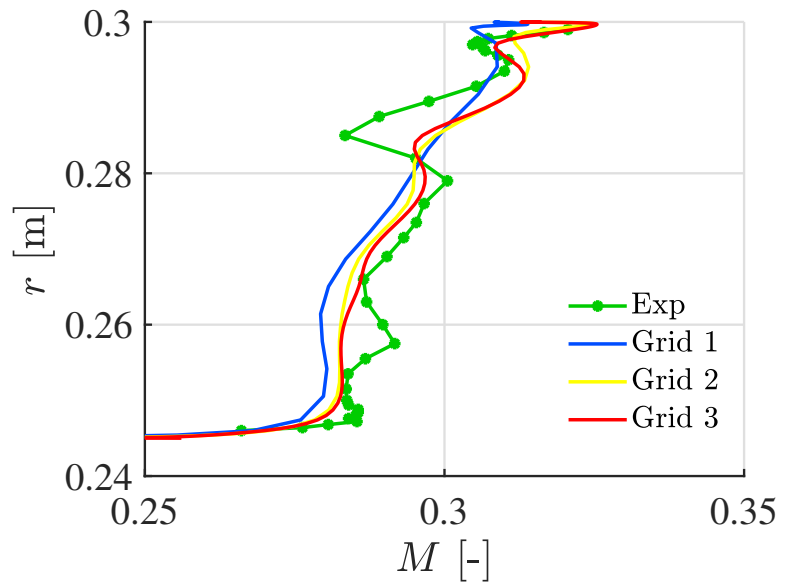

(b) S-EARSM model.

Fig. 13 Pitch average $M_{r, o u t, A}$ distribution. 


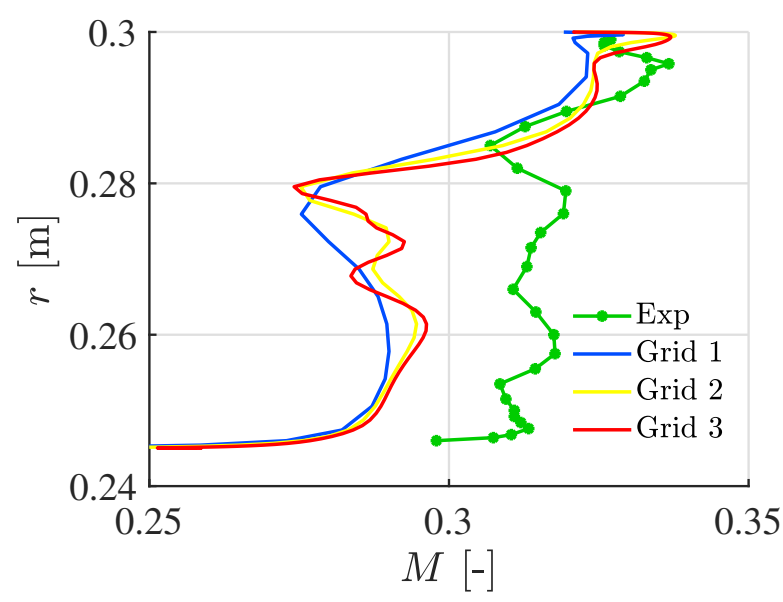

(a) SST model.

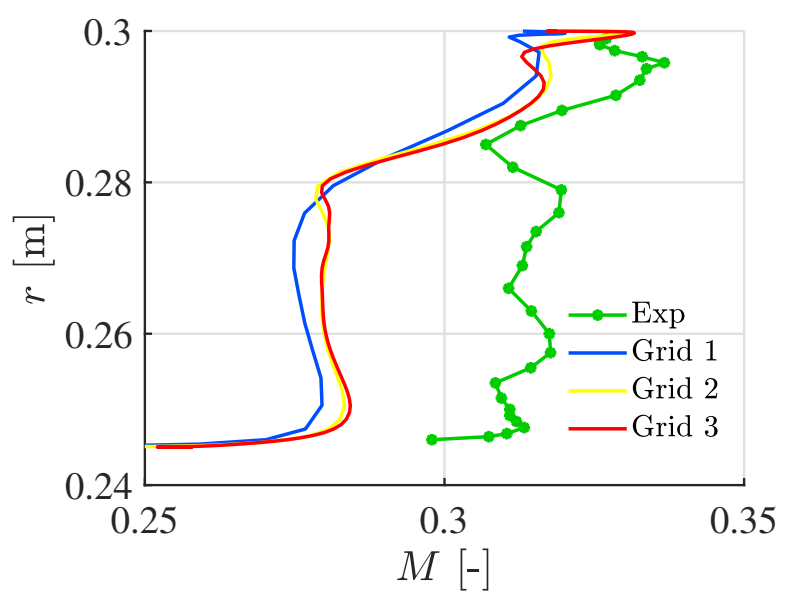

(b) S-EARSM model.

Fig. 14 Pitch average $M_{r, \text { out }, B}$ distribution.

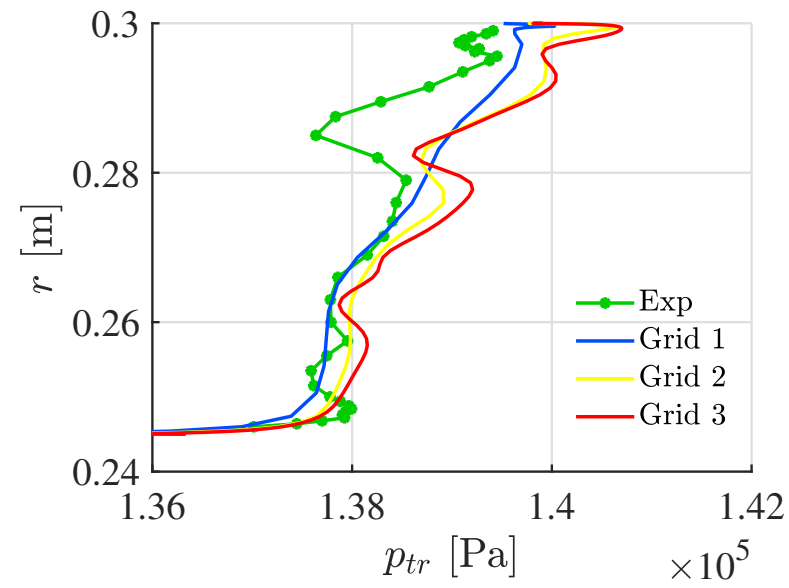

(a) SST model.

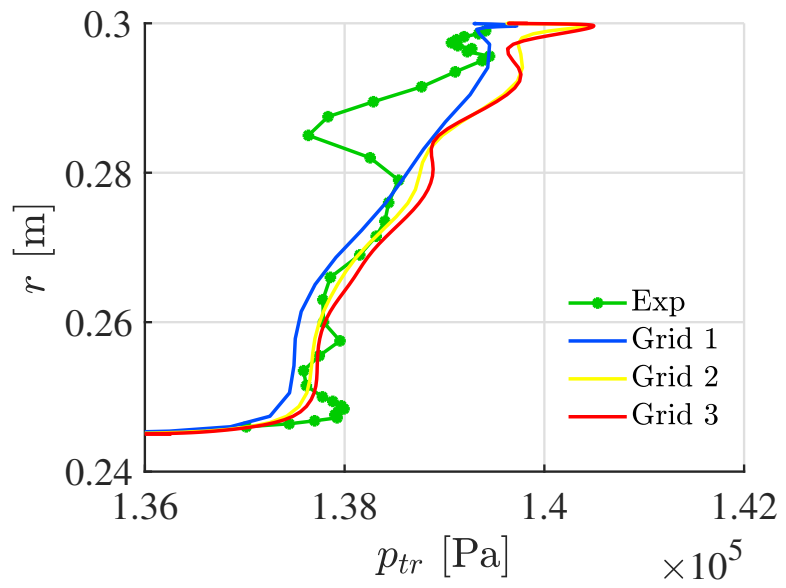

(b) S-EARSM model.

Fig. 15 Pitch average $p_{t r, o u t, A}$ distribution. 


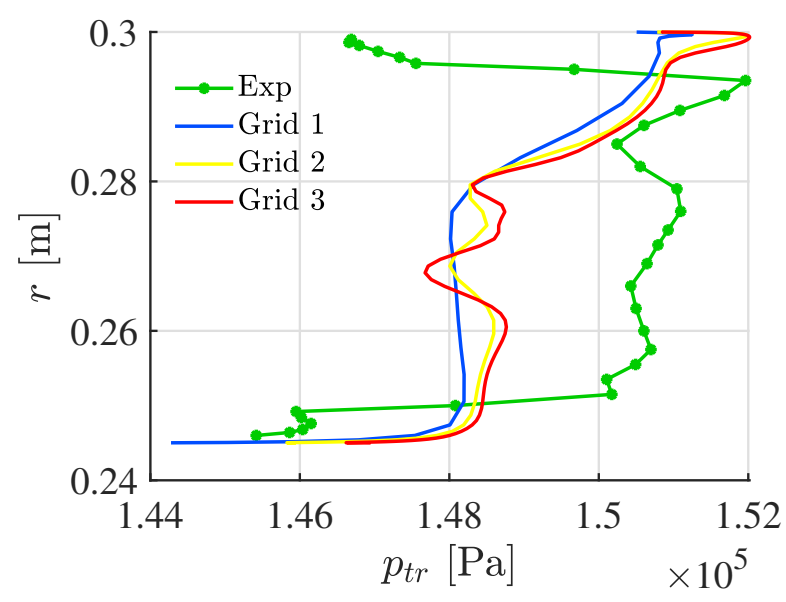

(a) SST model.

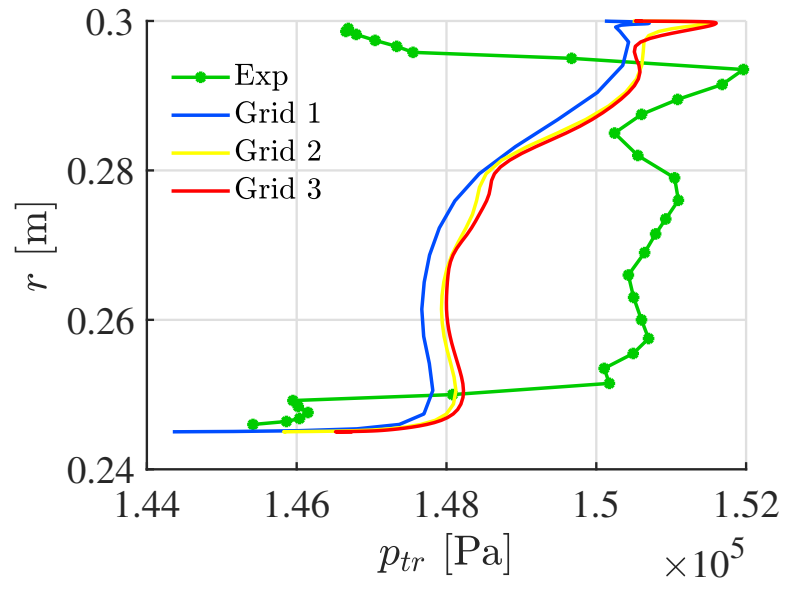

(b) S-EARSM model.

Fig. 16 Pitch average $p_{t r, \text { out }, B}$ distribution.

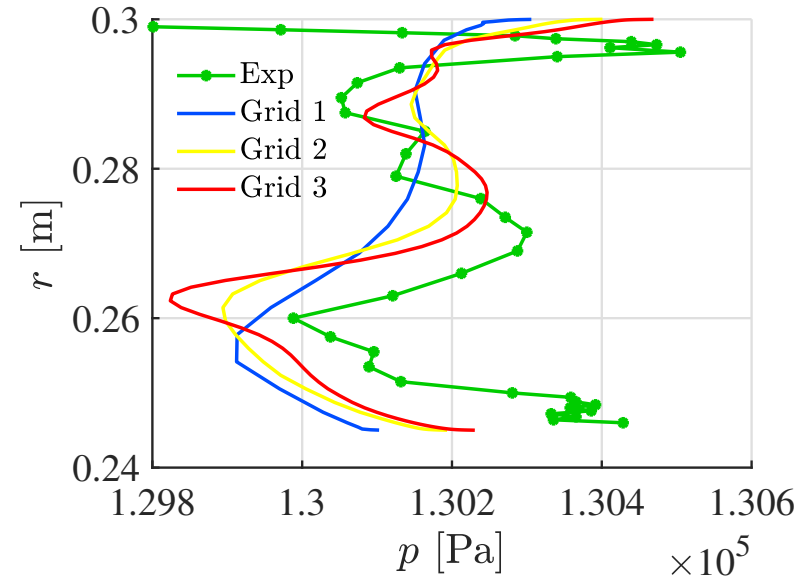

(a) SST model.

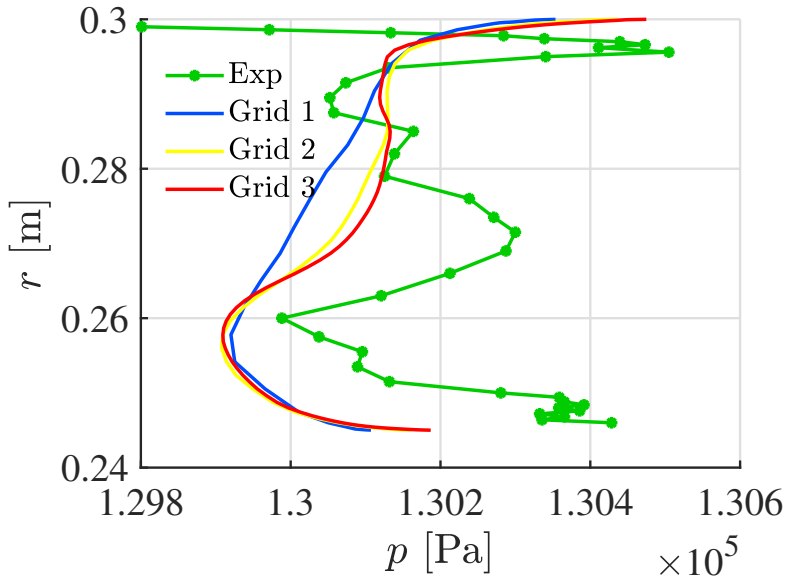

(b) S-EARSM model.

Fig. 17 Pitch average $p_{o u t, A}$ distribution. 


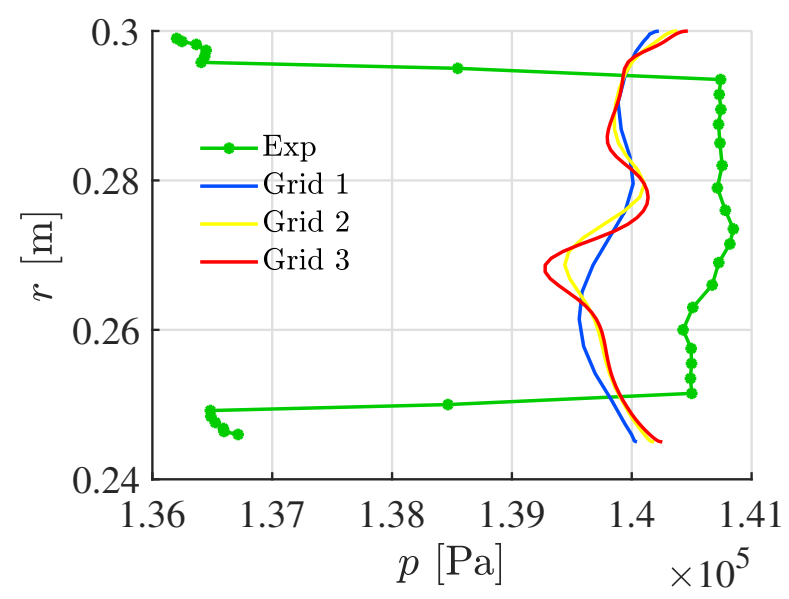

(a) SST model.

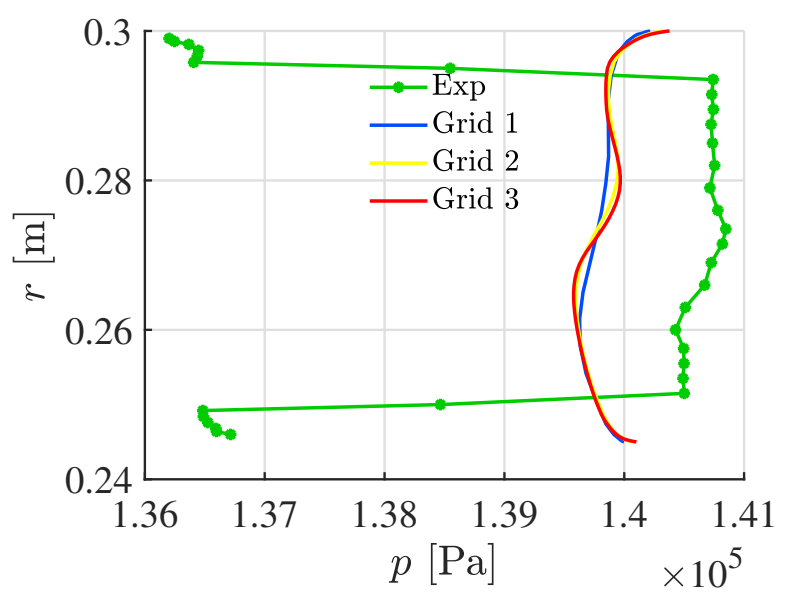

(b) S-EARSM model.

Fig. 18 Pitch average $p_{\text {out }, B}$ distribution. 